\title{
Impairment Models for Performance Evaluation of Digital Modulations in the Millimeter Wave Range
}

\author{
Dayan Adionel Guimarães, and Tiago Magalhães dos Reis
}

\begin{abstract}
The use of millimeter waves in wireless communication systems is expected to pave the way for solving the problem of spectrum scarcity currently experienced in lower frequencies, yet allowing for higher bit rates. However, impairments that are not of major concern in lower frequencies become relevant performance deterioration causes in the millimeter wave range, for instance the high signal attenuation and shadowing, as well as hardware-dependent impairments like phase noise, I-Q imbalance, and amplifier nonlinearities. In this tutorial article, the recent theory about these impairments are reviewed and applied to the development of models for assessing the performance of digital modulations in the millimeter wave range, under the separate and the joint effect of such impairments. The developed models are then used in the performance analysis of the $M$-ary frequency-shift keying ( $M$ FSK) modulation with non-coherent detection, and of the $M$-ary phase-shift keying (MPSK) and $M$ ary quadrature amplitude modulation (MQAM) with coherent detection. Simulation results bring out the different degrees of robustness of these modulations to the modeled impairments, highlighting the attractiveness of the MFSK and the 16QAM.
\end{abstract}

Index Terms-Amplifier nonlinearity, digital modulation, I-Q imbalance, millimeter wave, phase noise.

\section{INTRODUCTION}

$\mathbf{T}$ HE exponential growth in communications products and services experienced in the recent years is posing an unparalleled technological challenge to the development of new devices. Nonetheless, a given technology is not capable of supporting all the emerging needs, either due to its intrinsic hardware and systemic limitations, or due to the scarcity of some resource, for instance the radio-frequency (RF) spectrum.

It is estimated that in 2020 more than 50 billion devices will be connected through the fifth-generation $(5 \mathrm{G})$ data network [1]. In order to meet the demands for such a huge number of devices and higher data rates, $5 \mathrm{G}$ networks will probably take advantage of frequencies in the millimeter wave (mm-wave) range [2], i.e., $30-300 \mathrm{GHz}$, which is still mostly vacant.

Given the large bandwidth available, the use of mm-wave potentially improves data rates up to gigabits per second, which is comparable with optical fiber transmissions [3]. However, wireless communications using mm-wave need to overcome significant hurdles before they become mainstream. Among these hurdles, it can be mentioned high path loss,

D. A. Guimarães is with the National Institute of Telecommunications (Instituto Nacional de Telecomunicações, Inatel), Santa Rita do Sapucaí, MG, Brazil. T. M. dos Reis is with the Samsumg-Sidia, Manaus, AM, Brazil. Emails: dayan@inatel.br, tiago.r@samsung.com.

This work was partially supported by Finep, with Funttel resources, Grant 01.14.0231.00, under the Radiocommunications Reference Center (Centro de Referência em Radiocomunicações, CRR) project of the Inatel. DOI: 10.14209/jcis.2018.21. shadowing, and hardware-dependent impairments. Hence, the adoption of the aforementioned frequencies significantly reduces the coverage reach when compared to microwave links (3-30 GHz). In [3], the authors report examples of point-topoint wireless links in which a microwave system operating at $23 \mathrm{GHz}$ with the 256QAM modulation reached a distance of $3 \mathrm{~km} @ 1.4 \mathrm{Gbit} / \mathrm{s}$, while another system using 70/80 GHz spectrum reached up to $1.9 \mathrm{~km} @ 3 \mathrm{Gbit} / \mathrm{s}$ using the $2 \mathrm{PSK}$ modulation. Thus, in the mm-wave range, it is expected that even smaller distances will be covered.

\section{A. Related Work and Motivation}

The hardware development of devices aimed to be employed in lower frequencies is less critical than the one aimed at operating in higher frequencies. Hence, intrinsic hardware imperfections can limit system operation in the mm-wave range. As an example, phase noise, I-Q imbalance (or I-Q mismatch), amplifier nonlinearity, besides thermal noise, have to be addressed on models to be used in mm-wave system design and assessment [2]-[4]. Furthermore, attenuations caused by blockage of the electromagnetic wave in this frequency range, combined with multipath fading and shadowing, will result in a formidable challenge to the development of such models.

To achieve the high operating frequencies required by $\mathrm{mm}$ wave systems, a large frequency multiplication factor needs to be used in comparison to systems operating in lower frequencies, which results in larger oscillator phase noise variances. This large phase noise can become a bottleneck, since it causes rotation of the constellation symbols, affecting mainly the modulations with higher order [3].

On the other hand, it is known that relay stations are attractive solutions to extend the coverage of wireless systems by amplifying and retransmitting the incoming signals. Since it is desired that these stations have low-cost, their transceivers might have low quality, being prone to the above-mentioned impairments [1], [5]. For instance, in [5] the authors evaluate the impact of the I-Q imbalance and amplifier nonlinearity generated by relay transceivers. It is reported a performance ceiling whose effect becomes more pronounced as the quality of the components gets worse.

As far as the digital modulation schemes are concerned, it is likely that the well-known $M$-ary frequency-shift keying (MFSK), the $M$-ary phase-shift keying (MPSK) and the $M$ ary quadrature amplitude modulation (MQAM) will continue to be adopted in most of the upcoming systems. Contrasting with MPSK and MQAM, the increase of the modulation order $M$ for a fixed bit rate in $M$ FSK results in a wider 
bandwidth [6, pp. 432, 471]. Thus, the MFSK modulation is not a good choice to be used in frequency ranges that are already crowded. The application of the MFSK is nowadays restricted to narrowband systems, and to a few high capacity digital radio links. On the other hand, the use of the MFSK in the mm-wave range may be feasible due to the large amount of available spectrum.

Despite its low spectral efficiency, the MFSK modulation has a power efficiency that increases with $M$, that is, the bit error rate (BER) decreases as $M$ is increased, which is also opposite to what happens with MPSK and MQAM [6, p. 468]. Moreover, non-coherently detected MFSK has a reduced receiver complexity in comparison with coherent detection at a small performance penalty in terms of BER [6, p. 542], which makes it an attractive solution for low-cost devices in applications such as dense wireless sensor networks (WSNs) and the Internet of things (IoT). The performance gap between a coherently detected and a non-coherently detected MFSK signal may be even smaller if the inherent imperfection of the carrier recovery process for coherent detection $[6, \mathrm{Sec} .6 .8]$ is taken into account.

The MFSK modulation is also attractive for nonlinear amplification, since the modulated signal has an envelope that is almost constant after filtering [6, p. 508], allowing the use of high efficient amplifiers operating in the nonlinear region. Furthermore, this modulation is attractive for the implementation of direct conversion (zero-IF) receivers due to its robustness against DC-offset [7].

Recent research also consider the MFSK modulation as an interesting solution for millimeter wave communication systems, thanks to its claimed robustness against hardware and channel impairments [2], [8]. Particularly in [2], a simple hardware and channel impairment model is used to assess the performances of the modulations MFSK, MPSK and MQAM. Based on simulation results, a high emphasis is given to the robustness of the MFSK, with a large advantage over the other ones. However, this large advantage has come due to oversizing some impairments beyond the values that can be achieved with modern hardware architectures.

Thus, in light of the hardware and channel impairments that potentially will be faced by the wireless communication systems operating in high frequencies, especially in the millimeter wave range, it is of paramount importance to acquire the knowledge sufficient to understand, model and emulate such impairments, as well as the channel-related ones, aiming at giving adequate support to the research, design and assessment of digital communication systems. The present tutorial is an attempt to help the reader to acquire such a knowledge.

\section{B. Contributions and Organization of the Article}

In this tutorial, which expands [9], a survey on the background and models related to the most influential hardware and channel impairments that degrade the performance of wireless communications systems in high frequencies is presented. This survey aggregates pieces of information on the subject, which are typically fragmented or hidden throughout the specialized literature. This background is applied to the development of models aimed at analyzing the performance of digital modulations operating in the millimeter wave range. The analysis is made under the effect of each impairment separately, and also in the more practical-appealing situation in which the joint effect of the impairments is considered. The developed models are carefully tuned in terms of practical parameter values that match the actual hardware technology stage. They are then applied to the performance analysis of the MFSK modulation with non-coherent detection, and to the MPSK and MQAM modulations with coherent detection, for $M=4,16$ and 64 .

Besides the didactic appeal of the material presented herein, which goes far beyond [9], several results and conclusions, reproduced from [9] as well as new ones, bring out the different degrees of robustness of the above modulations to the modeled impairments. Among other important conclusions, these results highlight that the MFSK is not always the unique robust solution, a conclusion that contrasts with recent claims in the literature. In some cases, the 16QAM modulation is attractive as well.

The remainder of this article is organized as follows. Section II provides a characterization of each hardware imperfection and its modeling, also taking into account the channel and thermal noise effects. The individual models are combined in Section III, yielding a single model that accounts for the joint influence of the impairments. Section IV discusses the effect of the I-Q mismatch and the amplifier nonlinearities when modeled together or separately. Numerical results and discussions comparing the performances of the aforementioned modulations techniques are presented in Section V. The conclusions and opportunities for further investigations are drawn in Section VI.

\section{BACKGROUND AND MODELING OF EACH IMPAIRMENT}

Phase noise, I-Q imbalance, amplifier nonlinearity, and thermal noise are typical impairments generated by communication transceivers. In this section, the theoretical basis about each of these impairments is established, followed by the corresponding discrete-time model whose construction was grounded on such basis.

Figure 1 shows a simplified block diagram of a digital communication system, highlighting where three main hardware impairments are generated. The total phase noise depends on the quality of oscillators and frequency multipliers. I-Q imbalance is produced by differences between the gains in the in-phase and quadrature branches of the transceivers, as well as non-perfectly orthogonal carriers. Nonlinearity is caused by the amplifiers operating at nonlinear region. Besides these impairments, extra ones may occur and degrade the communication quality. For example, digital-to-analog (DAC) and analog-to-digital (ADC) converters insert intrinsic errors, time decision deviations as result of oscillator instability increases the symbol error rate. Moreover, in wireless communications the channel acts as a huge degradation source, especially at high frequencies.

\section{A. Phase Noise}

Oscillators play an important role in communications systems. They are responsible for generating synchronization 


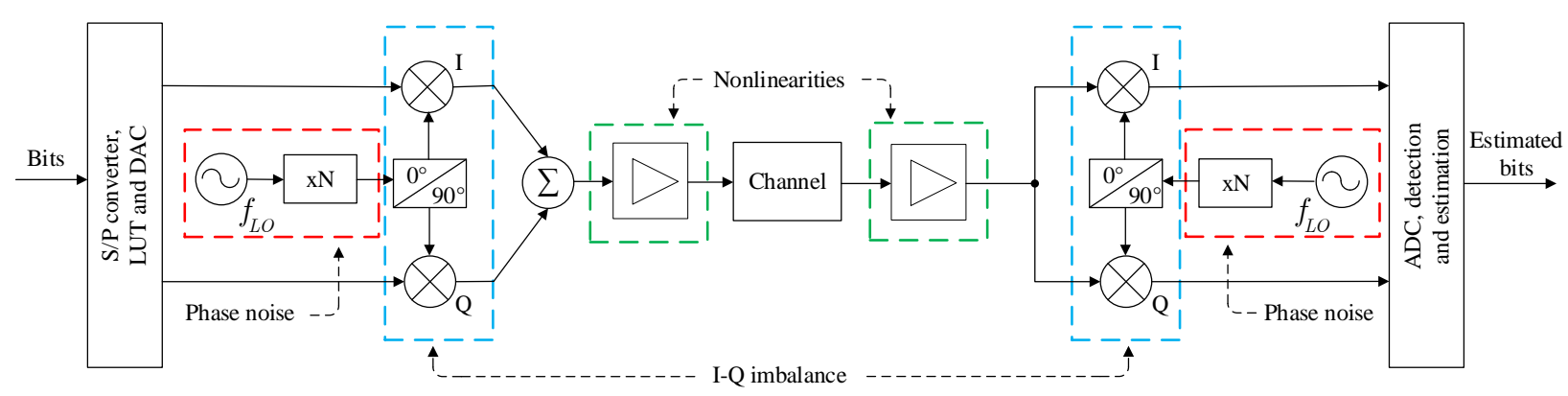

Fig. 1. Basic digital communication system example, highlighting three of the transceiver hardware impairment sources.

signals as well as up- and down-conversion carrier frequencies. In the case of carrier frequency generation, it is expected that the signal does not exhibit any fluctuation in amplitude or phase. In the frequency domain, it means that the signal should be a perfect Dirac delta function. However, in practice, due to hardware imperfection and the inherent thermal noise, the generated signal $V(t)$ always has amplitude and phase distortions that can be represented by [10]

$$
V(t)=[1+\xi(t)] \cos \left[2 \pi f_{c} t+\vartheta(t)\right],
$$

where $f_{c}$ is the carrier frequency, $\vartheta(t)$ models the phase fluctuations that produce the phase noise, and $\xi(t)$ models the amplitude fluctuations that are typically neglected in practice by using amplitude limiting mechanisms such as automatic gain control [10], [11].

As illustrated in Figure 2, the phase noise causes frequency deviations neighboring the center frequency. As the phase noise becomes higher, the side-lobes become wider and the peak amplitude at the center frequency reduces.

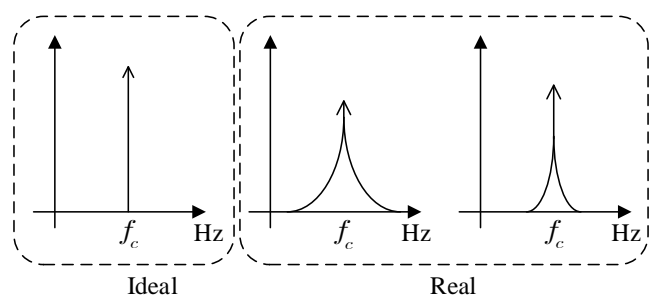

Fig. 2. Ideal and real carrier spectrum under different phase noise intensities.

In terms of modeling, let us assume that the phase noise intensity remains constant during a symbol interval $T$, which means that it can be considered a slow random process with respect to the symbol rate. In this case, the $n$-th sample at the receiver detector output, $r_{\mathrm{pn}}[n] \in \mathbb{C}$, under both phase noise and thermal noise influence, can be represented in the form

$$
r_{\mathrm{pn}}[n]=s[n] e^{j \phi[n]}+v[n],
$$

where $s[n] \in \mathbb{C}$ represents the received symbol, $\phi[n] \in \mathbb{R}$ is the phase noise sample, and $v[n] \sim \mathbb{C N}\left(0, N_{0} / 2\right)$ is the additive white Gaussian noise (AWGN) sample, with $N_{0}$ being the AWGN power spectral density (PSD). It is assumed that the AWGN is caused by thermal effect, so hereafter it is referred to as thermal noise [6, p. 79].
Figure 3 shows an ideal 16QAM constellation without thermal noise effect (left), and corrupted by phase noise (right). It can be seen that the phase noise effect is a random rotation of the symbol positions with respect to the center of the constellation. This rotation becomes more evident in symbols of higher energies. To plot this figure, it was considered a phase noise variance $\sigma_{\mathrm{ph}}^{2}=39 \times 10^{-5} \mathrm{rad}^{2}$, yielding an error vector magnitude (EVM) of $12.3 \%$. The EVM, to which a more formal treatment is devised in Section IV, is a measure of the dispersion of the generated constellation points with respect to their ideal positions. As a reference, the maximum allowable EVM for the LTE (Long Term Evolution) standard in the downlink is $12.5 \%$, according to the 3GPP (ThirdGeneration Partnership Program) [12, Sec. 14.3.4].
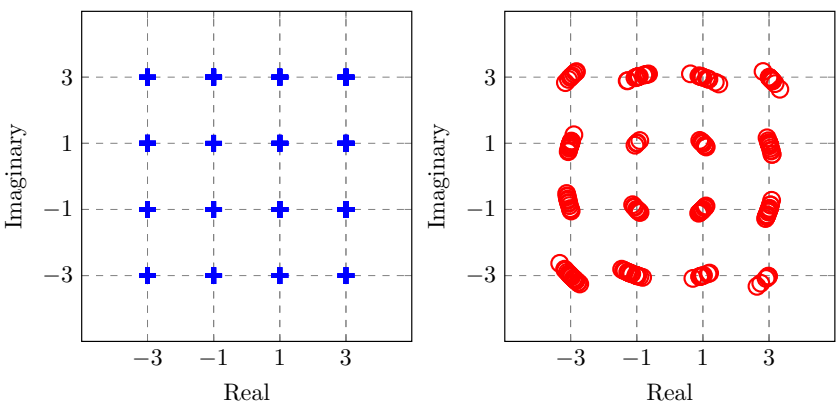

Fig. 3. Ideal 16QAM constellation (left) and corrupted by phase noise (right).

A well-known model for the phase noise consists in representing it as a Wiener process or a Brownian motion process [13]-[15], resulting in a phase noise PSD $S_{\phi}(f)$ whose intensity falls off as $1 / f^{2}$, or $-20 \mathrm{~dB} / \mathrm{dec}$. However, the phase noise PSD can exhibit other decay shapes, as illustrated in Figure 4. In this figure, three main shapes are shown in different spectral regions [10], [11]: the one with $-20 \mathrm{~dB} / \mathrm{dec}\left(1 / f^{2}\right)$, and other two with $-30 \mathrm{~dB} / \mathrm{dec}\left(1 / f^{3}\right)$ and $0 \mathrm{~dB} / \mathrm{dec}\left(1 / f^{0}\right)$. Systems with lower bandwidth, i.e. high symbol interval, will be more prone to the $1 / f^{3}$ and $1 / f^{2}$ phase noises. In the case of systems operating with higher bandwidths, as will probably happen in the mm-wave range, the $1 / f^{0}$ phase noise component becomes relevant as well. As an example, the authors in [16] assume the phase noise floor $\left(1 / f^{0}\right)$ as the main limiting factor.

From above, the total phase noise that must be taken into 


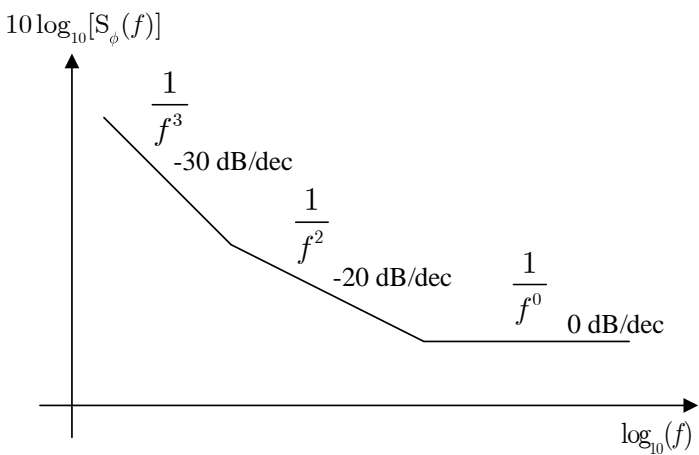

Fig. 4. Different phase noise PSDs in log-log scale.

account in (2) can be computed as

$$
\phi[n]=\phi_{0}[n]+\phi_{2}[n]+\phi_{3}[n]
$$

where the subscripts are associated to the slopes in Figure 4. In terms of simulations, $\phi[n]$ can be generated by filtering white Gaussian noise samples via low-pass filters whose transfer function in the Z-transform domain is [17]

$$
H_{\alpha}(z)=\frac{1}{\left(1-z^{-1}\right)^{\alpha / 2}},
$$

where $\alpha$ is the phase noise index $(0,2$ or 3$)$ and $w_{\alpha}[n]$ is a discrete-time white Gaussian noise $\mathcal{N}\left(0, \sigma_{\mathrm{ph} \alpha}^{2}\right)$. The block diagram of the resulting filter bank is illustrated in Figure 5. The variances $\sigma_{\mathrm{ph} \alpha}^{2}$ are given in Table $\mathrm{I}$, where $T$ is the sample interval (which is equal to the symbol interval), and the constant $K_{\alpha}$ controls the magnitude of the PSD in each segment, according to Figure 4.

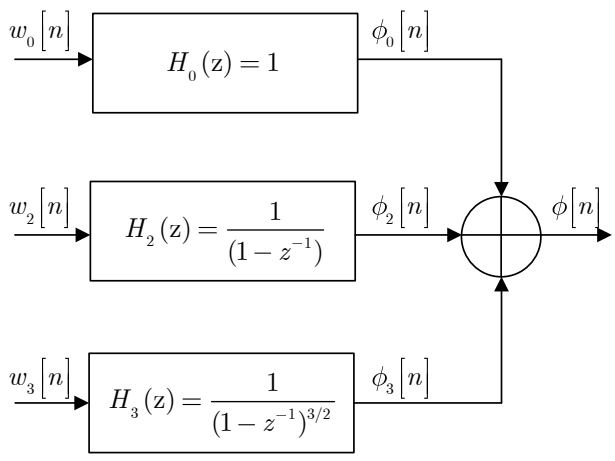

Fig. 5. Phase noise generator block diagram [10].

TABLE I

VARIANCES OF THE INPUT NOISES DEPICTED IN FIGURE 5 [10]

\begin{tabular}{c|c|c}
\hline Phase noise type & PSD & Input variance \\
\hline \hline$\phi_{0}[n]$ & $K_{0}$ & $\sigma_{\mathrm{ph} 0}^{2}=K_{0} T$ \\
\hline$\phi_{2}[n]$ & $K_{2} / f^{2}$ & $\sigma_{\mathrm{ph} 2}^{2}=4 K_{2} T \pi^{2}$ \\
\hline$\phi_{3}[n]$ & $K_{3} / f^{3}$ & $\sigma_{\mathrm{ph} 3}^{2}=8 K_{3} T^{2} \pi^{3}$ \\
\hline
\end{tabular}

\section{B. I-Q Imbalance}

Mobile systems have a severe physical space constraint and, hence, the radio-frequency stage has to be carefully designed. Applied along the years, superheterodyne receivers have high sensibility and selectivity, although employing more components when compared with direct conversion receivers (also called Zero-IF receivers) [18]. Zero-IF receivers down-convert radio-frequency directly to baseband. Figure 6 illustrates these two receiver structures.

The main advantage of Zero-IF receivers, besides the fact of employing a smaller amount of components, is image frequency cancellation through I-Q demodulation [18]. However, Zero-IF receivers are prone to I-Q imbalance, to DC offset due to self-mixing, to power leakage in full-duplex transceivers, and to flicker noise of shape $1 / f$ [4].

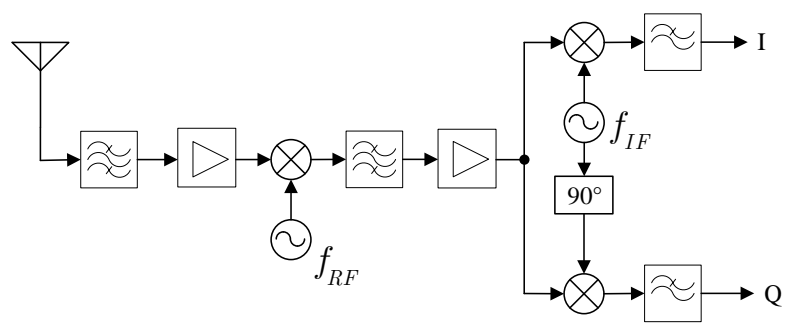

(a)

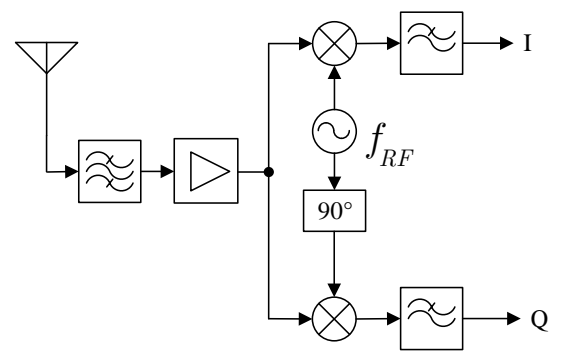

(b)

Fig. 6. Superheterodyne receiver (a), and Zero-IF receiver (b).

In practice, a real I-Q modulator does not produce a perfect orthogonality between in phase and quadrature components. The imbalance can be modeled as amplitude and phase errors in a symmetrical or asymmetrical manner. The former splits the error by half and applies to both components (I and Q). The latter assumes in phase as a perfect component and applies the error only in the quadrature part. According to [4], the asymmetrical form can be expressed by $V_{I}(t)=\cos \left(2 \pi f_{c} t\right)$ and $V_{Q}(t)=\rho \sin \left(2 \pi f_{c} t+\theta\right)$, where $\rho$ and $\theta$ model amplitude and phase errors, respectively. In ideal conditions, $\rho=1$ and $\theta=0$. Therefore, considering a polar baseband signal representation [6, p. 148], $s(t)=A(t) e^{j \psi(t)}$, where $A(t)$ denotes the amplitude modulation component and $\psi(t)$ denotes the phase modulation component, the radio-frequency signal can be expressed as $s_{R F}(t)=2\left[\Re\{s(t)\} \cos \left(2 \pi f_{c} t\right)-\mathfrak{J}\{s(t)\} \rho \sin \left(2 \pi f_{c} t+\theta\right)\right]$.

Thus, in terms of modeling, the $n$-th sample at the detector output under influence of I-Q imbalance is given by [4]

$$
r_{\mathrm{iq}}[n]=C_{1} s[n]+C_{2} s^{*}[n]
$$


where $*$ denotes complex conjugate, and

$$
C_{1}=\frac{1+\rho e^{-j \theta}}{2}, \quad C_{2}=\frac{1-\rho e^{j \theta}}{2} .
$$

For the ideal case (no I-Q imbalance), $C_{1}=1$ and $C_{2}=0$ [19]. It is informative to mention that in [19] the authors consider symmetrical errors, but their analysis can be extended to asymmetrical errors as well. As one can notice in (5), the received signal consists of two parts: the desired one and an undesired complex-conjugated version of the desired one.

Figure 7 shows a 16QAM constellation distorted by I$\mathrm{Q}$ imbalance. Using amplitude and phase imbalance factors $\rho=0.55 \mathrm{~dB}$ and $\theta=5.5^{\circ}$, respectively, yields an EVM of $12 \%$. This imbalance causes a shift on the constellation points with respect to their ideal positions; this effect gets worse in symbols with higher energies. Notice that the corrupted constellation no more has a square shape.

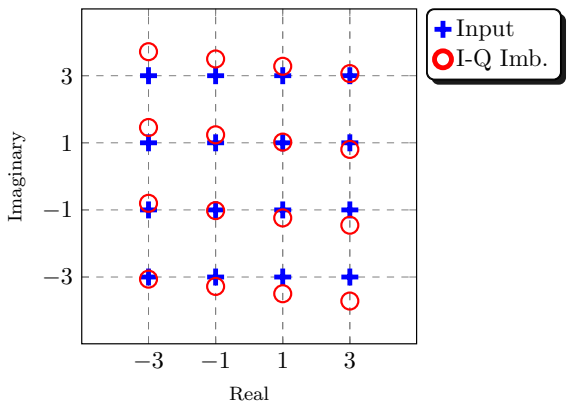

Fig. 7. Influence of the I-Q imbalance on a 16QAM constellation [4].

\section{Amplifier Nonlinearity}

In wireless communication systems, signal attenuation due to the free-space path loss at high frequencies occurs and amplifiers play an import role in order to compensate for such loss. Both fixed and variable-gain (as part of automatic gain control loops) amplifiers have this role.

A higher received signal power means, in general, a better communication quality in terms of BER. Two important parameters associated with the received signal power are the signal-to-noise ratio (SNR) and the ratio between the average received bit energy and the noise power spectral density, $E_{b} / N_{0}$, which are related through

$$
\frac{E_{b}}{N_{0}}=\frac{P_{\mathrm{rx}}}{N_{B}} \frac{B}{R_{b}}=\operatorname{SNR} \frac{B}{R_{b}},
$$

where $P_{\mathrm{rx}}$ is the average received signal power, in watts, $B$ the system bandwidth, in Hertz, $N_{B}$ is the noise power in this bandwidth, in Hertz, and $R_{b}$ is the bit rate, in bits per second.

While setting the received signal within a desired level or dynamic range, the amplifiers also introduce noise, which ends up reducing the SNR and the $E_{b} / N_{0}$. Nonetheless, in terms of modeling, this SNR reduction can be easily captured by an increased thermal noise power.

High levels of the input signal may push the amplifier towards a nonlinear working region, producing signal clipping [4, Sec. 6.5.1], thereby causing spectral regrowth [6, p. 511]. The clipping effect is especially degrading in the case of modulations that carry information based on amplitude levels, since it will limit the maximum allowable amplitude. On the other hand, the operation in the linear region requires a reduction of the input signal level by means of an input back-off (IBO). However, this process is power inefficient, since the device will be constantly operating under a regime of high power dissipation. In battery-powered devices, this operation regime drastically reduces the battery lifetime. The linear operating region of an amplifier typically corresponds to an input signal level about $1 / 3$ of the saturation amplitude.

The amplifier nonlinearity is mainly harmful in systems with non-constant envelope signals, for instance MQAM signals. Although MPSK signals have constant envelope, after filtering they will exhibit non-constant envelope [6]. According to [4], power amplifiers are the major sources of nonlinearities in a whole communication system.

In order to address the amplifier nonlinear effect, a polar baseband signal representation $s(t)=A(t) e^{j \psi(t)}$ is assumed. After nonlinear amplification, the resultant signal can be expressed in the time domain as $r_{\mathrm{a}}(t)=G_{A}[A(t)] e^{j\left\{\psi(t)+G_{\psi}[A(t)]\right\}}[4$, p. 188]. In the discretetime notation adopted herein, the amplified signal can be modeled as

$$
r_{\mathrm{a}}[n]=G_{A}(|s[n]|) e^{j\left\{\psi[n]+G_{\psi}(|s[n]|)\right\}},
$$

where $G_{A}(\cdot)$ and $G_{\psi}(\cdot)$ are the amplifier transfer functions in terms of AM-AM (amplitude modulation - amplitude modulation) conversion and AM-PM (amplitude modulation - phase modulation) conversion, respectively [4], and $|s[n]|$ and $\psi[n]$ are the magnitude and angle of $s[n]$, respectively.

The AM-AM function describes the relationship between the amplitudes of the output and input signals. This relationship should ideally be represented by a straight line whose slope is associated to the amplifier gain.

A known model for a typical AM-AM transfer function [20, p. 440] adapted to the present discrete-time notation is

$$
G_{A}(|s[n]|)=\frac{g|s[n]|}{\left[1+\left(\frac{g|s[n]|}{V_{\text {sat }}}\right)^{2 p}\right]^{\frac{1}{2 p}}},
$$

where $g$ and $p$ are, respectively, the dimensionless amplifier gain and the transfer function smoothness factor, and $V_{\text {sat }}$ the input saturation amplitude, in volts.

Figure 8 shows some AM-AM transfer functions of an amplifier with gain $g=\sqrt{2}$, for $V_{\text {sat }}=4$ volts (solid lines) and $V_{\text {sat }}=10$ volts (dashed lines), for some multiples of $p=0.81$. A higher $p$ makes the curve move towards the ideal one, for low amplitude signals, up to the saturation. Hence, a smaller $p$ reduces the amplifier linear operation range.

The AM-PM transfer function describes how the signal phase will be distorted by the amplifier. It translates the input signal amplitude variations into variations in the output signal phase. Ideally, this function should be constant and as close as possible to zero in the whole amplifier operation range. Systems which carry information on phase deviations are especially prone to the AM-PM conversion effect. For solidstate amplifiers, this effect has a minor importance compared with the AM-AM conversion [21]. 


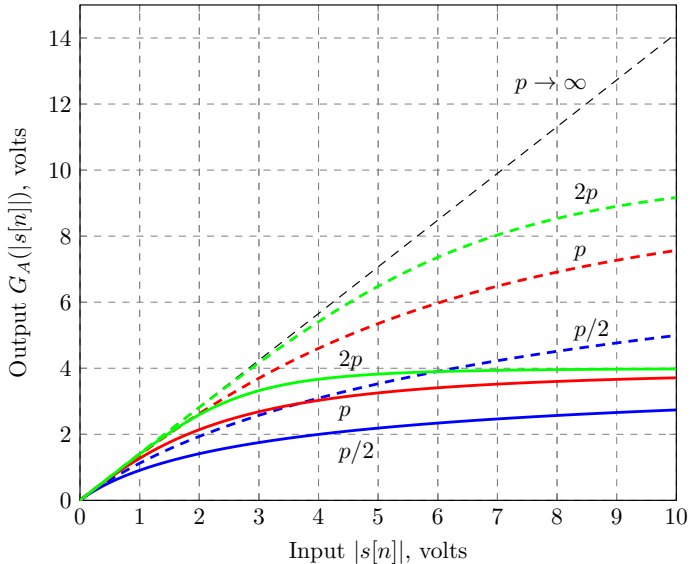

Fig. 8. AM-AM transfer functions of an amplifier with gain $g=\sqrt{2}$, for $V_{\text {sat }}=4$ (solid lines) and $V_{\text {sat }}=10$ (dashed lines), with $p=0.81$.

A known model for a typical AM-PM transfer function [20, p. 440] adapted to the discrete-time notation is

$$
G_{\psi}(|s[n]|)=\frac{\delta|s[n]|^{q_{1}}}{1+\left[\frac{|s[n]|}{\beta}\right]^{q_{2}}},
$$

where $\delta, q_{1}, q_{2}$, and $\beta$ are curve fitting parameters empirically determined from measurements on a real amplifier [20].

Figure 9 depicts AM-PM transfer functions of an amplifier, for different values of $\delta$ in (10), considering two transistor technologies at $60 \mathrm{GHz}$ : CMOS (Complementary Metal-oxide Semiconductor) and GaAs (Gallium Arsenide). Typical parameters were adopted to plot this figure [20]: The CMOS technology (dashed lines) considers $\delta=2560, \beta=0.114$, $q_{1}=2.4$, and $q_{2}=2.3$; the GaAs technology (solid lines) considers $\delta=-48000, \beta=0.123, q_{1}=3.8$, and $q_{2}=3.7$. It can be seen that larger values of $\delta$ increase the phase shift of the output signal; the two technologies produce opposite shifts for a given $\delta$, with slightly smaller values in favor of the CMOS. Moreover, it can be observed in Figure 9 that the phase shift variations with the input signal amplitude become smaller beyond $|s[n]|>0.5$ volts; small values of $\delta$ correspond to smaller variations.

Figure 10 illustrates what happens to a 16QAM constellation when going through an amplifier with GaAs technology due to the AM-AM and AM-PM conversion effects. The parameters expressed in (9) and (10) were set to values of practical significance [20]: $g=\sqrt{2}, V_{\text {sat }}=6$ volts, $p=0.81$, $\delta=-48000 / 6, \beta=0.123, q_{1}=3.8$, and $q_{2}=3.7$. The input constellation had its energy changed until the output EVM was about $12.5 \%$ in order to meet the LTE specification [12, Sec. 14.3.4]. This EVM happened for an input constellation energy of about 2.3 joules.

The AM-AM effect makes high energy symbols less amplified compared with low energy symbols, i.e., there is a gain compression. The higher the difference between low and high amplitude levels, more pronounced becomes the distortion of the constellation shape. As can be noticed in Figure 10, this shape tends to a circumference.

The AM-PM effect can be observed in Figure 10 as a clockwise rotation about the constellation center (if the

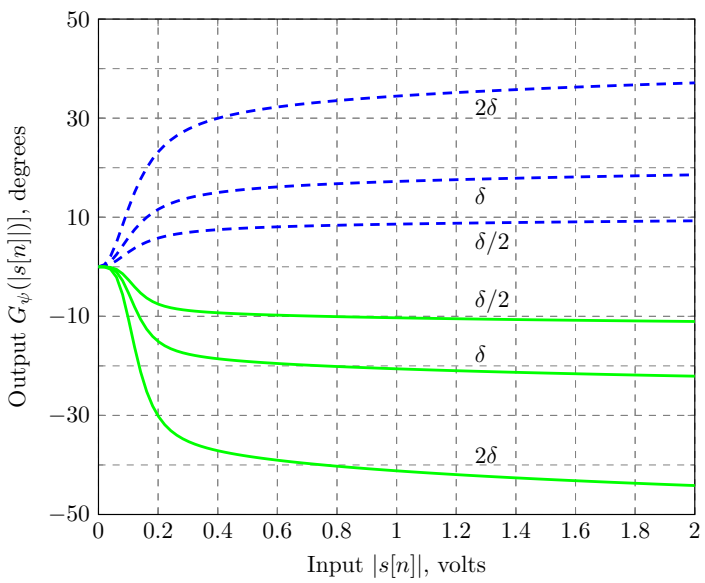

Fig. 9. AM-PM transfer functions of an amplifier, for different values of $\delta$ in (10), considering two transistor technologies at $60 \mathrm{GHz}$ : CMOS (dashed lines) and GaAs (solid lines).

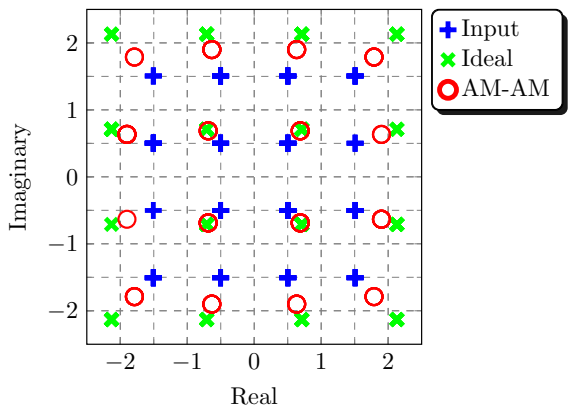

(a)

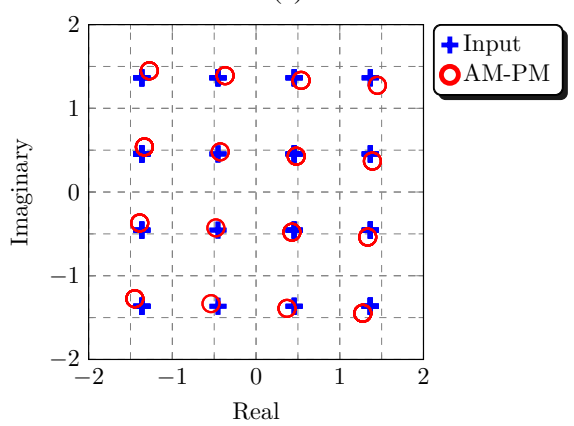

(b)

Fig. 10. 16QAM constellations under nonlinear amplifier effects: (a) AM-AM conversion (b) AM-PM conversion.

CMOS technology were considered, the rotation would be counterclockwise). Considering the same constellation energy employed in the AM-AM effect analysis, the resultant AMPM EVM was approximately $6 \%$. This indicates that the phase rotation effect might be less intense than the one observed on the signal amplitude.

Observing (8)-(10) and Figures 8-10, one can infer that modulations in which the information is not carried on the signal amplitude or phase variations, which is the case of frequency-shift keying signals, can be less impaired by the amplifier nonlinear effects. As a consequence, the power efficiency can be raised [22].

It is informative to mention a specific model for the nonlinear effects of an amplifier, as given in [23]. In this 
model, under certain circumstances the output of a nonlinear device can be modeled by a smaller copy of the input signal plus an error given by an independent zero-mean Gaussian random variable. In other words, the nonlinear effects are jointly represented by random perturbations of the original symbols. Other models go beyond and combine the effects of the amplifier nonlinearity and the I-Q imbalance into a single Gaussian random variable, for instance the ones considered in [2], [4], [5]. As demonstrated later on in this article, the fusion of impairments into a single one may result in inaccurate models and results, thus leading to erroneous interpretations or conclusions.

\section{Channel Impairments}

A line-of-sight (LOS) channel under influence of multipath and shadowing is adopted in this work, assuming a flat and slow fading so that the multiplicative fading channel model [6, p. 213] can be applied. Therewith, the received signal $r_{\mathrm{ch}}(t)$ is represented by the multiplication of the transmitted signal $s(t)$ by the channel gain $h \in \mathbb{C}$, in baseband representation, that is, $r_{\mathrm{ch}}(t)=h \times s(t)$. Under the vector channel model, it can be written that

$$
r_{\mathrm{ch}}[n]=h \times s[n]
$$

where $h$ is the multiplicative shadowed fading given by [2]

$$
h=\sqrt{\frac{L\left(d_{0}\right)}{X}\left(\frac{d_{0}}{d}\right)^{\gamma}}\left(h_{\operatorname{los}} \sqrt{\frac{\mathcal{K}}{1+\mathcal{K}}}+h_{\mathrm{nlos}} \sqrt{\frac{1}{1+\mathcal{K}}}\right),
$$

where the term inside the square root on the left accounts for the area mean power loss, which is distance-dependent according to the Log-distance model [6, p. 201], combined with the local mean power variation about the area mean that occurs due to shadowing [6, p. 202]. The quantity $L\left(d_{0}\right)=\left(\lambda / 4 \pi d_{0}\right)^{2}$ is the inverse free-space path loss at the reference distance $d_{0}$, where $\lambda=c / f_{c}$ denotes the carrier wavelength, with $c$ and $f_{c}$ as the speed of the light in vacuum in meters per second, and the carrier frequency in hertz, respectively. The quantity $X$ is a lognormal random variable that accounts for the shadowing. In logarithmic scale, it is a zero mean Gaussian random variable with standard deviation $\sigma_{\mathrm{sh}}$, meaning that the local mean received power wanders about the area mean with a Gaussian distribution having a standard deviation of $\sigma_{\mathrm{sh}} \mathrm{dB}$. The distance between transmitter and receiver is denoted by $d$, and $\gamma$ is the path loss exponent, which is an environmentdependent quantity [6, p. 202].

A Rician multipath fading is modeled by the right-hand side term between parentheses in (12). The factor $h_{\mathrm{los}}=e^{j \pi \sin \varrho}$ represents the line-of-sight complex channel gain, with $\varrho$ being the signal angle of arrival at the receiver (notice that the line-of-sight magnitude gain is unitary, and that only a phase rotation is imposed to the LOS signal component). The nonline-of-sight (NLOS) component, $h_{\text {nlos }} \sim \mathbb{C N}(0,1)$, models the multipath fading. The ratio between the average power of the dominant LOS component and the variance of the remaining multipath NLOS ones is given by the Rice factor $\mathcal{K}[6$, p. 212], expressed in linear scale in (12).

\section{Model FOR THE JoInt EFFECT OF CHANNEL AND HARDWARE IMPAIRMENTS}

Following the received signal path in Fig. 1, the joint effect of the impairments discussed in the previous section can be modeled by properly combining the individual models defined in (2), (5), (8) and (11). It is firstly assumed that the received symbol is impaired by the amplifier nonlinearity, then by the I-Q imbalance, subsequently by the phase noise, and lastly by the thermal noise. Since the channel gain is assumed constant during the symbol interval, its multiplicative effect can be shifted to act on the signal resultant of the joint effect of the amplifier nonlinearity and the I-Q imbalance. Thus, the $n$-th complex sample at the detector output under the joint effect of all impairments described in Section II can be expressed as

$$
r_{\mathrm{comb}}[n]=h\left(C_{1} r_{\mathrm{a}}[n]+C_{2} r_{\mathrm{a}}^{*}[n]\right) e^{j \phi[n]}+v[n],
$$

where the nonlinear-distorted signal $r_{\mathrm{a}}[n]$ results from the action of (8) on the constellation symbol $s[n]$. Subsequently, the I-Q imbalance model (5) is applied to the input sample $r_{\mathrm{a}}[n]$. The result is operated via the phase noise model (2), having the nonlinear-distorted and I-Q imbalanced signal as input. The result of this operation is multiplied by the complex channel gain according to (11), and finally added to the thermal noise component $v[n]$.

It is worth emphasizing that the model (13) improves the one presented in [2] by modeling the nonlinear effects separated from the I-Q imbalance, and by adding the phase noise components $1 / f^{3}$ and $1 / f^{0}$ that form $\phi[n]$ via (3), to the single component $1 / f^{2}$ taken into account in [2]. One must recall that each hardware impairment modifies the position of the constellation points. However, each one acts in their own way, motivating the above-mentioned separation. The impact of separating or not the nonlinear effects from the I-Q imbalance is further explored in Section IV.

It is also important to bear in mind that the model (13) and the one given in [2] are vector models that can be directly applied to MPSK or MQAM receivers with coherent detection [6, pp. 427-440]. For non-coherently detected MFSK [6, p. 537] signals, an adaptation must be performed so that the phase noise standard deviation becomes proportional to the frequency employed by each symbol. In other terms, the model described in (13) cannot be directly applied to the noncoherently detected MFSK modulation because such technique does not has a discrete signal space representation equivalent to the MPSK and MQAM signals. This is owed the fact that the phase of the carrier is time-variant with respect to the carrier used at the transmitter, meaning that the signal space representation is a continuously rotating point [6, p. 507].

One way of representing an MFSK signal using a signal space is presented in [24, Sec. 10.2]. As an example, Figure 11 shows an MFSK symbol tone been represented by eight complex samples. As a matter of fact, the MATLAB software processes an MFSK signal, as detected by the non-coherent receiver given in [6, Fig. 6.84], by means of a sequence of samples likewise those shown in Figure 11.

Thus, the model (13) is directly applicable to the samples at the output of a quadrature detector when MPSK or MQAM 


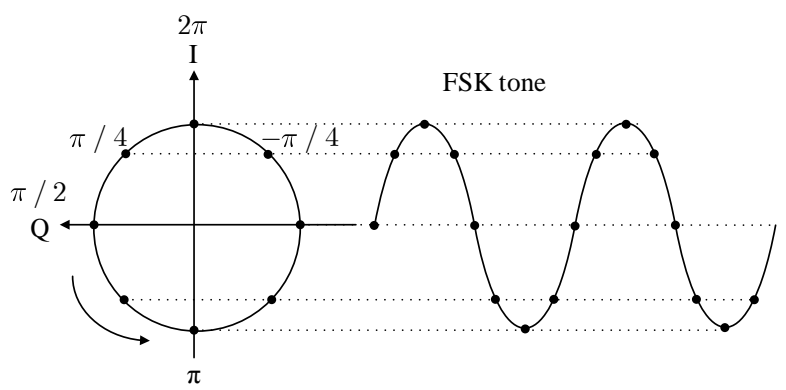

Fig. 11. A sequence of complex numbers representing an MFSK tone (right) and its signal space representation (left).

modulations with coherent detection are considered. In the case of non-coherently detected MFSK, an adaptation must be done to account for the particularities of the phase noise. If the phase noise is a slow process (much slower than the symbol rate), the same phase noise sample must be used in all intrasymbol samples. If the phase noise is fast when compared with the symbol rate, different phase noise samples must be adopted for each intra-symbol sample. In what concerns the other impairments considered by the present model, they are equally applied to all intra-symbol samples, since they are assumed to be constant within the symbol interval.

\section{IMPACT OF COMbining THE I-Q ImbaLANCE AND THE AMPLIFIER NONLINEARITY IN A SINGLE IMPAIRMENT}

In [2], [4], and [5] the authors model the I-Q imbalance and the amplifier nonlinearity as a unique complex Gaussian random variable $\eta \sim \mathbb{C N}\left(0, \sigma_{\mathrm{hw}}^{2}\right)$ that is added to a version of the original signal, where $\sigma_{\mathrm{hw}}^{2}$ characterizes the intensity of these imperfections together. Obviously, this model is simple to implement; its accurateness, though, needs to be quantified.

This section presents an analysis of the impact of splitting or not the I-Q imbalance and the amplifier nonlinearity. This analysis starts with the definition of the percentage root mean square (rms) EVM [25], which can be computed via

$$
\mathrm{EVM} \%=\sqrt{\frac{\frac{1}{N_{s}} \sum_{n=0}^{N_{s}-1} I_{\mathrm{e}}[n]^{2}+Q_{\mathrm{e}}[n]^{2}}{\frac{1}{M} \sum_{m=0}^{M-1} I_{\mathrm{r}}[m]^{2}+Q_{\mathrm{r}}[m]^{2}}} \times 100 \%,
$$

where the errors $I_{\mathrm{e}}[n]$ and $Q_{\mathrm{e}}[n]$ respectively denote the inphase and the quadrature difference between the measured and the reference symbol sample, that is, $I_{\mathrm{e}}[n]=I_{\mathrm{m}}[n]-I_{\mathrm{r}}[n]$ and $Q_{\mathrm{e}}[n]=Q_{\mathrm{m}}[n]-Q_{\mathrm{r}}[n]$, where the subscripts "m" and "r" denote the measured and the reference quantities, respectively. The parameter $N_{s}$ is the number of received symbols used in the computations and $M$ is the modulation order. Thus, the numerator indicates the average error power and the denominator indicates the average constellation power.

Table II shows the range of parameter values employed in this EVM analysis, whose first set of results are plotted in Figure 12. Each point on this figure was obtained from $2 \times 10^{8}$ symbols by computing the EVM in MPSK and $M Q A M$ modulations under the presence of thermal noise with $E_{b} / N_{0}=26 \mathrm{~dB}$. This $E_{b} / N_{0}$ value will be adopted hereafter when the AWGN noise is not the target variable for analysis, aiming at facilitating the comparisons with the results from [2]. Assuming the use of the GaAs technology, the other parameters were assumed to be $\beta=0.123, q_{1}=3.8, q_{2}=3.7$, and a unitary amplifier gain. Each number on the horizontal axis of Figure 12 corresponds to a set of the four parameters extracted from those in Table II, which are $\left(\rho, \theta, V_{\text {sat }}\right.$, and $\delta)$. As an example, number 1 corresponds to the set $(0.9 \mathrm{~dB}$, $\left.9^{\circ}, 2 \mathrm{~V},-48000 / 2\right)$, number 2 refers to the set $\left(0.8 \mathrm{~dB}, 8^{\circ}\right.$, $3 \mathrm{~V},-48000 / 3)$, number 3 corresponds to $\left(0.7 \mathrm{~dB}, 7^{\circ}, 4 \mathrm{~V}\right.$, $-48000 / 4)$, and so on. Thus, the leftmost numbers represent low quality hardware, whereas the rightmost ones represent higher quality hardware. As expected, poorer hardware produces higher EVMs. Moreover, as one can see in Figure 12, there is an error floor starting to show up for high-quality devices, and it is caused by the prevalence of the thermal noise with respect to the other impairments.

TABLE II

RANGE OF VALUES FOR IMPAIRMENT ANALYSIS.

\begin{tabular}{l|c}
\hline Parameters & Range \\
\hline \hline I-Q Imbalance $(\rho$ and $\theta)$ & $0.9 \mathrm{~dB}$ to $0.05 \mathrm{~dB}$ and $9^{\circ}$ to $0.5^{\circ}$ \\
Nonlinearity $\left(V_{\text {sat }}\right.$ and $\left.\delta\right)$ & $2 \mathrm{~V}$ to $11 \mathrm{~V}$ and -24000 to -4364
\end{tabular}

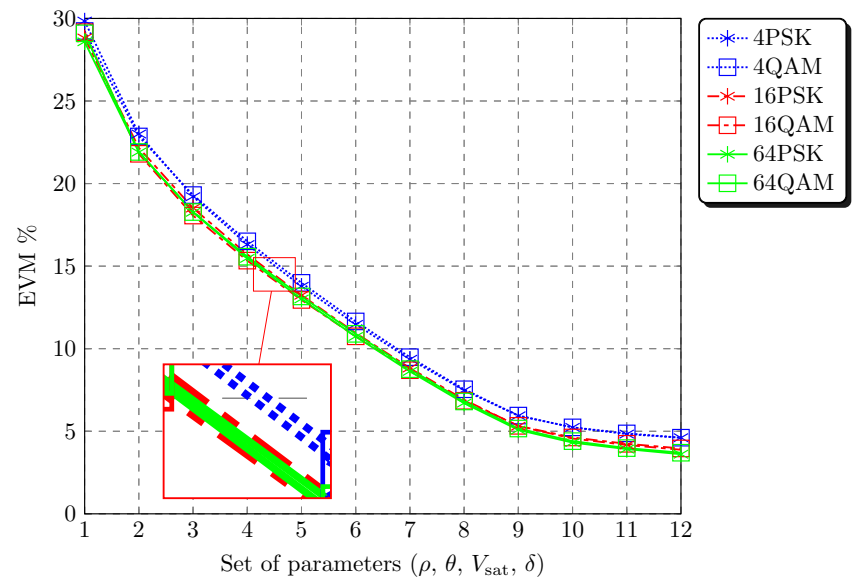

Fig. 12. Final EVM from I-Q imbalance and amplifier nonlinearity separately modeled. The zoomed region makes it easier to identify the relative curve positions.

Since (14) defines an rms EVM, the argument of the square root can be seen as the variance $\sigma_{\mathrm{hw}}^{2}$ of a random variable $\eta$ that, in principle, can be used to model the combined effect of several impairments. In order to verify the accurateness of modeling the I-Q imbalance and amplifier nonlinearity as a unique impairment, as considered in [2], [4], [5], in this section the variance $\sigma_{\mathrm{hw}}^{2}$ is used to parametrize the generation of a zero-mean complex Gaussian random variable to directly impair the constellation symbols.

Figure 13 shows the ratio between the EVMs obtained from the two approaches: i) I-Q imbalance and amplifier nonlinearity combined in a single random variate $\eta$, and ii) I-Q imbalance and amplifier nonlinearity modeled separately. The numbers on the horizontal axis represent the same sets of parameters adopted in the case of Figure 12. It can be noticed 
from this figure that the EVMs produced by the variable $\eta$ always exceed the ones resulted from the I-Q imbalance and the amplifier nonlinearity when modeled separately. This behavior is even more pronounced when high-quality hardware is employed, i.e, for sets of parameters indexed by the higher numbers on the horizontal axis of Figure 13. The important conclusion drawn from this experiment is that a single complex Gaussian random variable as defined in the case of $\eta$ shall not be applied to model the combined effect of the I-Q imbalance and the amplifier nonlinearity, because it always results in a higher EVM. Nonetheless, if the variance of $\eta$ is calculated in such a way to reduce the error between the EVMs produced under the above-described approaches, it can be used to model the I-Q imbalance and the amplifier nonlinearity together. In this, case, the model simplification aimed at with the use of $\eta$ is somewhat blurred by the complexity of generating it with the correct variance.

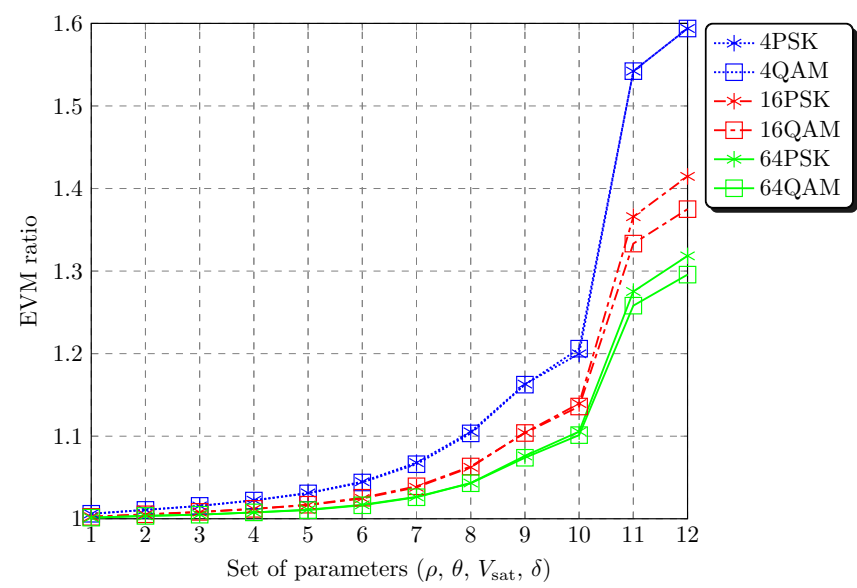

Fig. 13. Ratio between the EVMs coming from the combined effect of the I-Q imbalance and amplifier nonlinearity simulated by the random variable $\eta$, and the ones coming from the separate effect of these impairments.

\section{NUMERICAL RESUlTS AND DisCUSSIONS}

In this section, simulation results regarding the effect of hardware and channel impairments on the coherently detected $M$ PSK and MQAM modulations, and on the non-coherently detected MFSK modulation, for $M=4,16$ and 64, are presented and discussed.

The operation frequency and path loss exponent $\gamma$ can be any; here they were chosen as $60 \mathrm{GHz}$ and 4, respectively, without loss of generality. The reference distance and the distance between the transmitter and receiver were set to $d_{0}=1 \mathrm{~m}$ and $d=25 \mathrm{~m}$, respectively. The Rice factor was assumed to be $\mathcal{K}=5 \mathrm{~dB}$ and the arrival angle of the LOS component was drawn from a uniform distribution, i.e. $\varrho \sim \mathcal{U}[0,2 \pi)$. Each point on the BER curves shown hereafter was obtained via $2 \times 10^{5}$ Monte Carlo simulations with each simulation performed over a frame length of 100 symbols.

As a preliminary result, Figure 14 shows the performances (BER versus $E_{b} / N_{0}$ ) of the MPSK and MQAM modulations for the parameters pertaining to the set number 5 adopted to plot Figures 12 and 13, which are $\rho=0.5 \mathrm{~dB}, \theta=5^{\circ}$,
TABLE III

RANGES OF PARAMETERS ADOPTED IN THE SIMULATIONS

\begin{tabular}{l|c}
\hline Parameter & Range \\
\hline \hline Total phase noise $\left(\sigma_{\mathrm{ph}}^{2}\right)$ & $1.032 \times 10^{-6}$ to $1.032 \times 10^{-3}$ \\
I-Q imbalance $(\rho, \theta)$ & 0.05 to $1 \mathrm{~dB}, 0.5^{\circ}$ to $10^{\circ}$ \\
Amplifier nonlinearity $\left(V_{\mathrm{sat}}, \delta\right)$ & 2 to $11 \mathrm{~V},-24000$ to -4364 \\
Shadowing standard deviation $\left(\sigma_{\mathrm{sh}}\right)$ & 6 to $12 \mathrm{~dB}$ \\
Path loss exponent $(\gamma)$ & 3 to 5 \\
Average received SNR per bit $\left(E_{b} / N_{0}\right)$ & 1 to $31 \mathrm{~dB}$ \\
\hline
\end{tabular}

$V_{\text {sat }}=6$ volts, and $\delta=-48000 / 6$. This set was chosen because it lies approximately in the middle of the sets considered in those figures. Moreover, notice from Figure 13 that this set corresponds to a relatively small difference between the two modeling approaches.

The lines in Figure 14 correspond to the effects of the IQ imbalance and amplifier nonlinearity impairments modeled individually, and the markers are associated with the approach of using the random variable $\eta$ to model the combined impairments. It can be observed that, for $M=4$, the model that uses $\eta$ is well applicable to the whole range of $E_{b} / N_{0}$. However, when $M$ increases beyond 4, the performance with $\eta$ exhibits an error floor, whereas the real performance gets better with higher values of $E_{b} / N_{0}$. When $M=64$, the error floor appears for both approaches, indicating that for high modulation orders the hardware employed must have a better quality. By doing so, the performance will be less affected by adopting any of the impairment modeling approaches, and the BER curve will tend to the theoretical performance over a flat and slow Ricean fading channel.

From above, the most important conclusion is that modeling the I-Q imbalance and the amplifier nonlinearity as a unique complex Gaussian variable $\eta$ is only applicable for highquality hardware, a situation in which the performance is less influenced by these impairments. The approach of using $\eta$ is only suitable for lower modulation order $M$, according to Figure 14. A higher $M$ requires more carefully-designed hardware, making it possible to exchange the individual impairments modeling approach by the joint one via $\eta$. Finally, it is important to highlight that modeling the above impairments with a unique complex Gaussian variable always will produce an error floor, but this floor can occur for impractical (very high) values of $E_{b} / N_{0}$, and may not be even noticed if the system is not assessed in these high $E_{b} / N_{0}$ values.

Table III shows the ranges of values used to obtain the simulation results shown from this point on. These ranges were selected from a bunch of experimentations or theoretical analyses reported in [2], [5], [15], [16], [26], [27] and [28], and were chosen to allow for comparisons between results reported in these references and those presented hereafter.

\section{A. Effect of the Phase Noise}

In [15], the authors propose a lower bound on the innovation process related to the Brownian motion adopted to model the phase noise with PSD shape $1 / f^{2}$. This bound is frequency- 


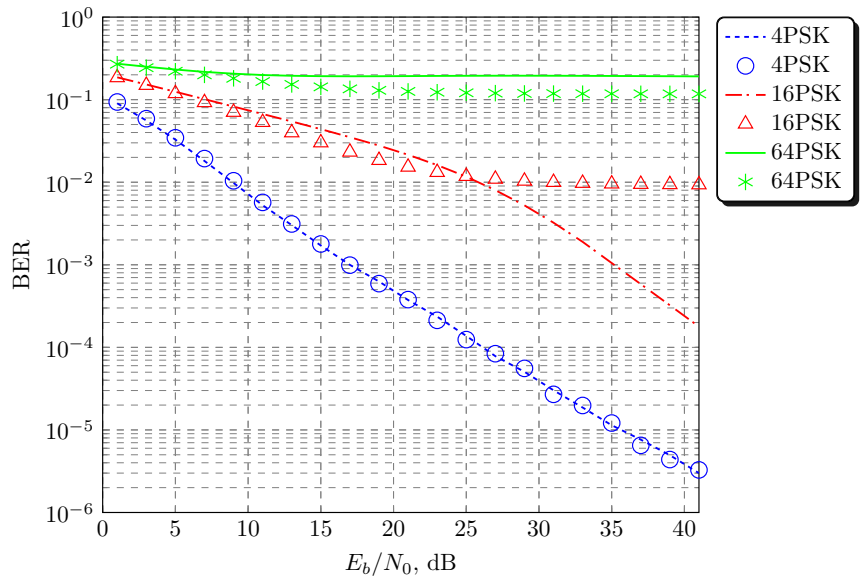

(a)

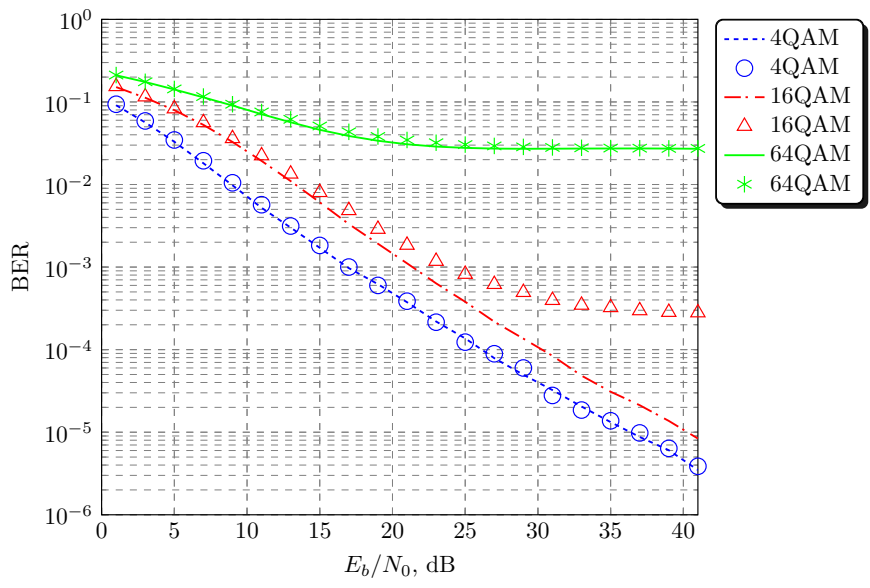

(b)

Fig. 14. BER versus $E_{b} / N_{0}$ for the two impairment approaches (individually and via $\eta$ ): (a) $M$ PSK and (b) $M$ QAM, for $M=4,16$ and 64 . Lines correspond to the effects of the I-Q imbalance and amplifier nonlinearity modeled individually; markers are associated to the use of $\eta$.

dependent and technology-dependent, yielding a phase noise variance limited according to

$$
\sigma_{\mathrm{ph} 2}^{2} \geq \frac{19.496 \times 10^{-21} \pi^{2} f_{c}{ }^{2}}{I_{d} V_{d} Q_{0}^{2} B}
$$

where, for an oscillator design, $I_{d}$ and $V_{d}$ denote the transistor collector-drain operating current and safe operating voltage, respectively, $Q_{0}$ is the unloaded quality factor of the resonator, and $B$ is the system bandwidth. Noteworthy that the safe operating voltage is normally about $1 / 3$ of the input saturation point of the device. Using the GaAs HEMT (high-electronmobility transistor) technology [15] with $f_{c}=60 \mathrm{GHz}$ and $B=1 \mathrm{MHz}$ and safe operating voltage, one can obtain the $1 / f^{2}$ phase noise variance $\sigma_{\mathrm{ph} 2}^{2} \approx 1.3 \times 10^{-5} \mathrm{rad}^{2}$.

Aiming at mimicking a reduction of the hardware design complexity, the total phase noise variance has been increased until the EVM reached $8 \%$, for $E_{b} / N_{0}=26 \mathrm{~dB}$. This EVM corresponds the maximum allowable value for the 64QAM modulation in the LTE downlink [12, Sec. 14.3.4]. The resultant variance was found to be $\sigma_{\mathrm{ph}}^{2}=\sigma_{\mathrm{ph} 0}^{2}+\sigma_{\mathrm{ph} 2}^{2}+\sigma_{\mathrm{ph} 3}^{2} \approx 13.411 \times 10^{-5} \mathrm{rad}^{2}$. To compute the variances of the phase noise components $1 / f^{0}$ and $1 / f^{3}$, which are $\sigma_{\mathrm{ph} 0}^{2}$ and $\sigma_{\mathrm{ph} 3}^{2}$, respectively, it was applied the relation between them and the variance of the noise component $1 / f^{2}$, according with Kasdin's technique [17] and Table I, for $K_{0}=-110 \mathrm{~dB}, K_{2}=10$, and $K_{3}=10^{4}$.

Figure 15 shows the performances of the modulations under analysis for this fixed phase noise variance $\sigma_{\mathrm{ph}}^{2}=13.411 \times 10^{-5}$ $\mathrm{rad}^{2}$. As one can notice from this figure, the BER attained by the 16PSK and the 16QAM modulations exhibit error floors around $1 \times 10^{-2}$ and $5 \times 10^{-4}$, respectively. The error floors in the 64PSK and 64QAM modulations are around $1 \times 10^{-1}$ and $1.6 \times 10^{-2}$, respectively. The 4PSK(4QAM) and MFSK modulations for all $M$ did not unveil any error floor in the investigated $E_{b} / N_{0}$ range. One must be aware of the unexpected poor performance of the 64QAM, in spite of the compliance with the maximum EVM established by the 3GPP standard for the LTE downlink. However, the specified maximum EVM corresponds to an acceptable constellation quality over the AWGN channel [16]; here it was adopted a Ricean fading channel, yielding useless BER values even for such EVM. The same reasoning applies to the interpretation of the results regarding to the 16QAM modulation.

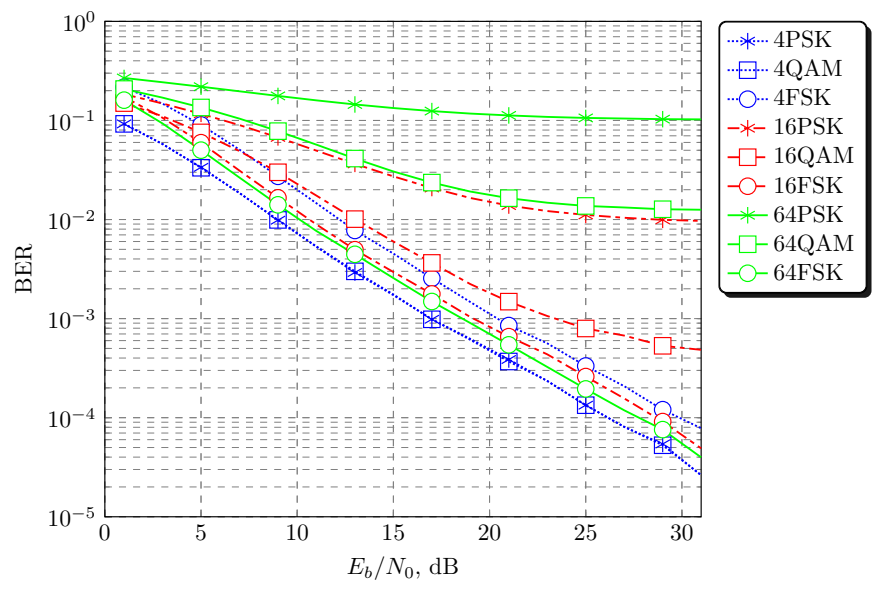

Fig. 15. BER versus $E_{b} / N_{0}$ for a total phase noise variance $\sigma_{\mathrm{ph}}^{2}=13.411 \times 10^{-5} \mathrm{rad}^{2}$

Figure 16 shows the performances of the modulations, for $E_{b} / N_{0}=26 \mathrm{~dB}$, under different levels of the total phase noise variance. As expected, the MPSK and the MQAM modulations exhibit a performance degradation as the phase noise variance increases, whereas the MFSK modulations remain with the same performance in the whole range of analysis. Higher oder MPSK and MQAM modulations are less robust against the phase noise level variation, with the $M$ PSK being less robust than the MQAM.

It is important to highlight that, for extremely high phase noise variances, the performance of the MFSK modulations may display an unexpected BER behavior when changing the modulation order. For instance, a case study is reported in [2], in which the BER increase as $M$ increases at very high phase noise levels. Fortunately, as already pointed out in the present article, such high phase noise levels are not realistic at present technology development stage. In other words, the hardware quality nowadays is capable of producing a phase noise with 


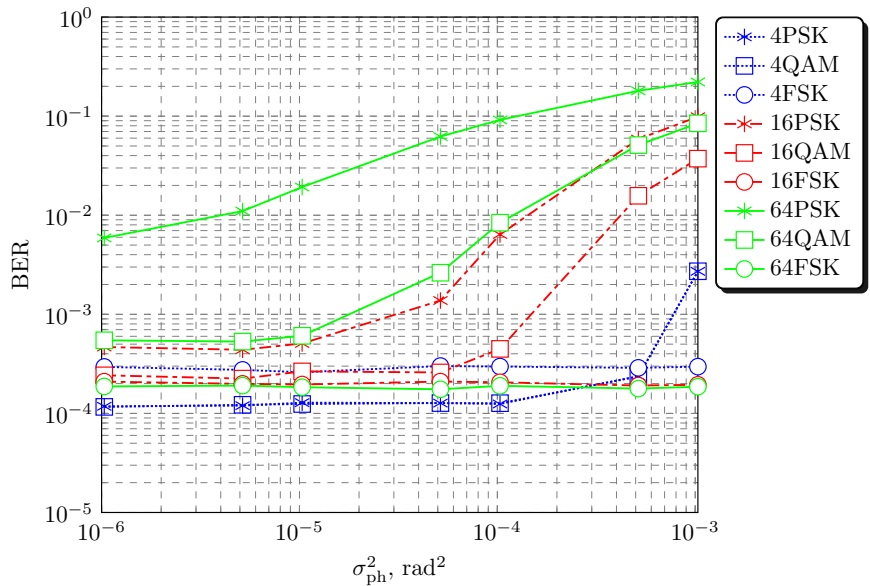

Fig. 16. BER versus $\sigma_{\mathrm{ph}}^{2}$, for $E_{b} / N_{0}=26 \mathrm{~dB}$.

variance far below those critically high levels adopted in [2].

The BER growth as $M$ increases in the MFSK modulations can be explained in light of Figure 4. Low order modulations, which correspond to narrower bandwidths, typically will be mostly influenced by the $1 / f^{3}$ phase noise component, whilst high order modulations, which correspond to wider bandwidths, may be additionally impaired by the $1 / f^{2}$ and $1 / f^{0}$ components. Then, for a given phase noise variance, it means that higher effective phase noise levels will contribute to the performance degradation as $M$ increases. At some high phase noise level, these new components may produce a performance degradation higher than the improvement that would be brought by the larger modulation order, reverting the BER variation with respect to $M$.

\section{B. Effect of the I-Q Imbalance}

The range of values in Table III for the I-Q imbalance was defined based on real measurements reported in [16], and theoretically assessed in [27]. In [16], the authors consider a communication testbed implemented to operate in the millimeter wave range, with symbol rates 5,10 , and $20 \times 10^{9}$ symbols per second, modifying the amplitude and phase difference between the in-phase and quadrature components. Following the same methodology used in the phase noise analysis, the values for $\rho$ and $\theta$, as defined in Section II-B, were empirically determined to yield an EVM of $8 \%$ for the 64QAM modulation, considering $E_{b} / N_{0}=26 \mathrm{~dB}$.

Figure 17 presents the performances of the modulations for a fixed I-Q imbalance $\left(\rho=0.35 \mathrm{~dB}\right.$ and $\left.\theta=3.5^{\circ}\right)$. The I-Q imbalance has a smaller effect on the performance when compared with the phase noise. It can be seen in Figure 17 that only the 64PSK modulation exhibited an error floor over the analyzed range of $E_{b} / N_{0}$. The 4PSK/4QAM modulation unveiled the lowest BER, even when compared with the 64FSK modulation.

Figure 18 shows the BER versus the I-Q imbalance, for $\rho$ ranging from $0.05 \mathrm{~dB}$ to $1 \mathrm{~dB}$, and $\theta$ from $0.5^{\circ}$ to $10^{\circ}$, for $E_{b} / N_{0}=26 \mathrm{~dB}$. As expected, higher imbalances represent worse BERs, a behavior that becomes evident for $M>4$ in the

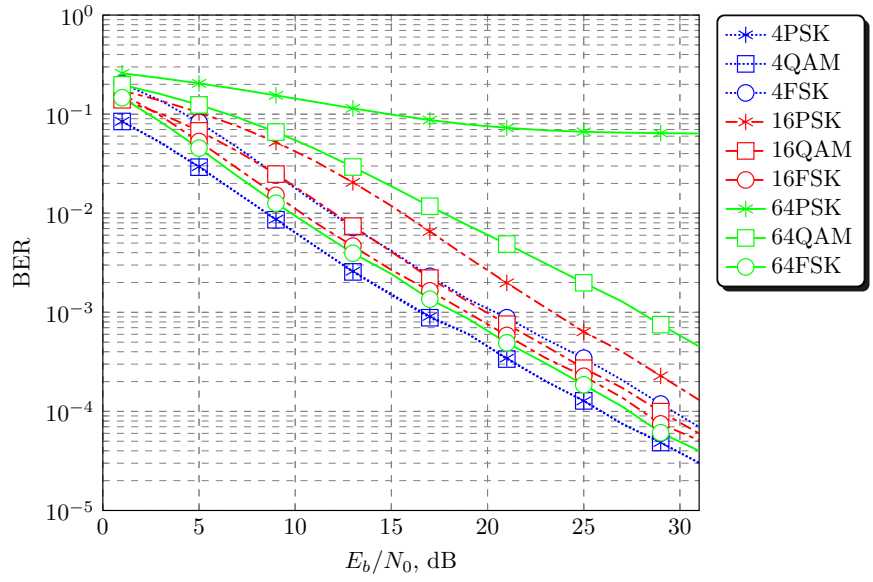

Fig. 17. BER versus $E_{b} / N_{0}$ with $\rho=0.35 \mathrm{~dB}$ and $\theta=3.5^{\circ}$.

cases of MPSK and MQAM modulations. It is interesting to notice that the 64QAM modulation outperformed the 16PSK modulation at $\rho=\theta / 10=0.9$. It can be seen that the MFSK modulations practically were not affected by the I-Q imbalance range analyzed, likewise in the case of the phase noise.

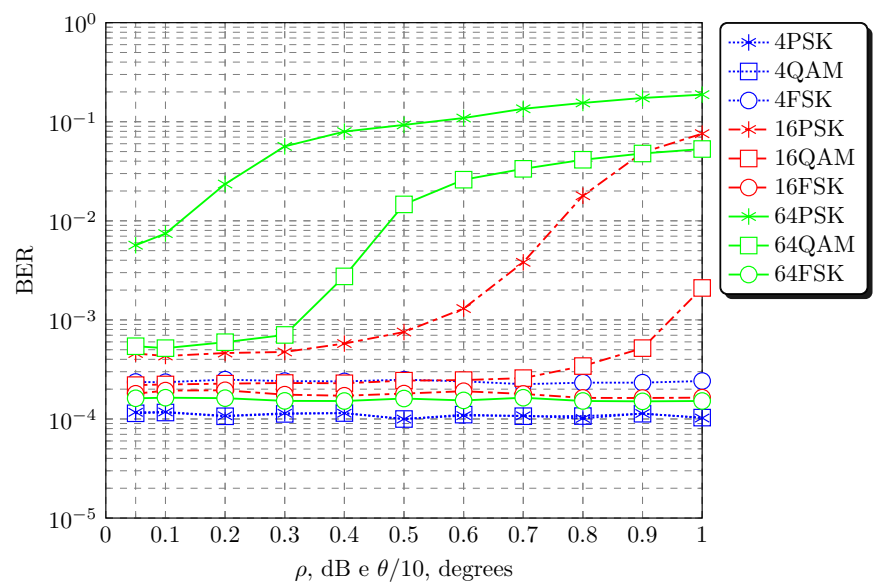

Fig. 18. BER versus $\rho$ and $\theta / 10$, for $E_{b} / N_{0}=26 \mathrm{~dB}$.

\section{Effect of the Amplifier Nonlinearity}

The range of values for amplifier nonlinearities given in Table III was based on the resultant EVM for each combination of $V_{\text {sat }}$ and $\delta$, using the 4PSK and 64QAM modulations as references. According to the LTE documentation [12], the EVM upper bounds for these modulations are $17.5 \%$ and $8 \%$, respectively. The range for $V_{\text {sat }}$ and $\delta$ in Table III yields EVM values that include those required by the standard, assuming $E_{b} / N_{0}=26 \mathrm{~dB}$ : For $V_{\text {sat }}=2$ volts and $\delta=-24000$, the resultant EVM is $23 \%$, whereas for $V_{\text {sat }}=11$ volts and $\delta=-4364$, the resultant EVM is $4.2 \%$. In this last point of the range of values for $V_{\text {sat }}$ and $\delta$, the resultant EVM, which is composed by the thermal noise and amplifier nonlinear effects, is such that the thermal noise influence cannot be overlooked in comparison with the beginning of the range, where the thermal noise can be neglected due to the dominance of the amplifier nonlinear effect. 
An alternative approach could be used in order to address the amplifier nonlinearity influence. Instead of using $V_{\text {sat }}$ and $\delta$, one could resort to the IBO (input back-off). The IBO is the difference between the input power that would produce the maximum output power, relative to the actual input power applied to the amplifier. For instance, if an input power of $-25 \mathrm{dBm}$ produces the maximum output power, and the actual input power is $-30 \mathrm{dBm}$, the IBO is $5 \mathrm{~dB}$. Hence, a higher IBO should be used if it is desired that the amplifier operates towards its linear region. On the other hand, it means a lower power efficiency. In this article, the $V_{\text {sat }}$ and $\delta$ were adopted due to the higher degree of freedom in modeling the amplifier nonlinear effects.

Figure 19 was constructed following the same methodology applied to the analysis of phase noise and I-Q imbalance. It shows BER results versus $E_{b} / N_{0}$, assuming a fixed impairment $\left(V_{\text {sat }}=6\right.$ volts and $\left.\delta=-8000\right)$, and an EVM of $8 \%$. Similar to the I-Q imbalance (see Figure 17), the amplifier nonlinearity is less harmful when compared with the phase noise (see Figure 15). It can be seen that an error floor occurred only for the 64PSK modulation over the range of $E_{b} / N_{0}$ values analyzed. The other modulations monotonically produce a reduction of the BER as $E_{b} / N_{0}$ increases. The lowest BER values are attained by the 4PSK/4QAM modulation, closely followed by the MFSK family and the 16QAM.

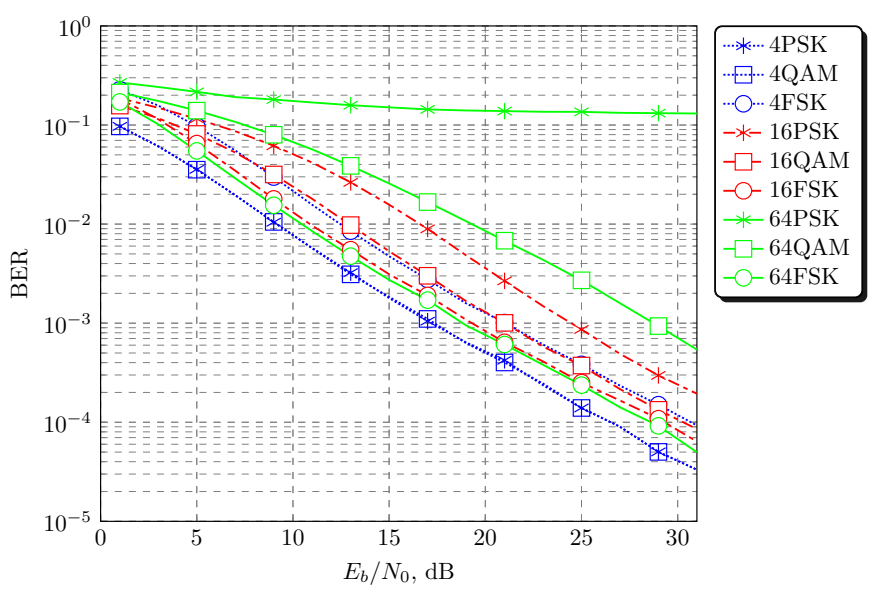

Fig. 19. BER versus $E_{b} / N_{0}$, for $V_{\text {sat }}=6$ volts and $\delta=-6000$.

Figure 20 allows for a BER analysis in terms of modifying $V_{\text {sat }}$ and $-\delta / V_{\text {sat }}$, with $V_{\text {sat }}$ varying from 2 to 11 volts and $\delta=-48000$, with $E_{b} / N_{0}=26 \mathrm{~dB}$ [20]. Regarding the remaining parameters in (9) and (10), were assumed $p=0.81$, $q_{1}=3.8, q_{2}=3.7$, and $\beta=0.123$, according to the GaAs pHEMT (pseudomorphic HEMT) technology [20, p. 441], with unitary amplifier gain, that is, $g=1$.

From Figure 20 it can be noticed that the systems suffer from a strong performance degradation at the lowest saturation point, $V_{\text {sat }}$, which corresponds to a strong nonlinear effect, except for the lower order modulations and all modulations of the family $M F S K$. In case of $V_{\text {sat }}>4$ volts, the influence of the nonlinearity has a minor impact on the BER for the 16PSK and 16QAM modulations. For the 64QAM, this behavior happens for $V_{\text {sat }}>7$ volts. In the case of the 64PSK modulation, it was not possible to determine such a point with the employed hardware parameters.

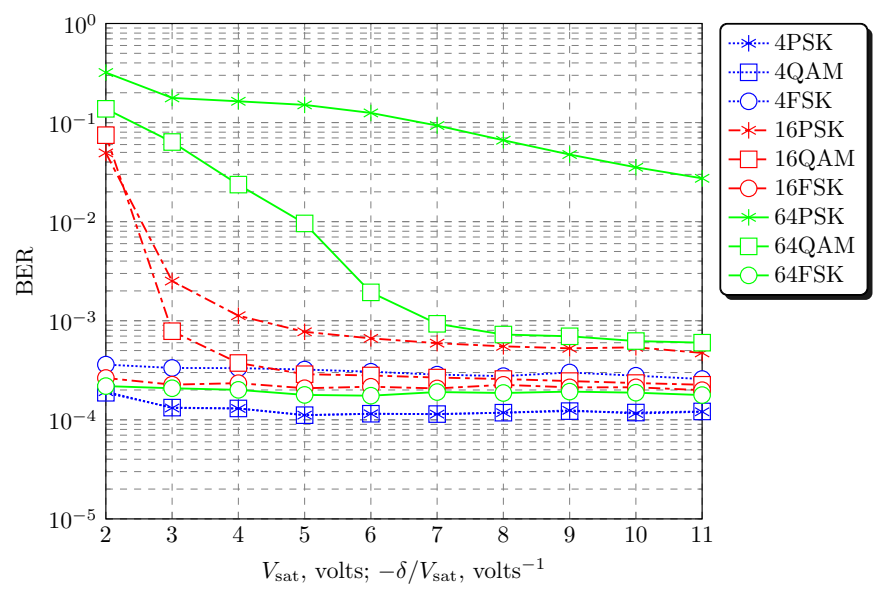

Fig. 20. BER versus $V_{\text {sat }}$ and $-\delta / V_{\text {sat }}$, for $E_{b} / N_{0}=26 \mathrm{~dB}$.

\section{Effect of the Channel Impairments}

The ranges of the parameters employed for channel modeling were based on [2], [26] and [28], over different system configuration. In [26] and [28] were reported experimental results for LOS links at $28 \mathrm{GHz}^{1}$ and $78 \mathrm{GHz}$. Path loss exponents around 1.7 and 1.6, and shadowing standard deviations around $2.5 \mathrm{~dB}$ and $3.2 \mathrm{~dB}$, respectively, were found. These values increase for NLOS links under different antenna polarizations, to 5.1 and 6.4 , and to $11.6 \mathrm{~dB}$ and $15.9 \mathrm{~dB}$, respectively. The ranges of channel-related parameters given in Table III are those adopted in [2], which encompass some of the above LOS and NLOS conditions over different antenna polarizations and carrier frequencies.

Figure 21 shows the BER values attained by the modulations $M F S K, M P S K$ and $M$ QAM, for $M=16$ and $M=64$, by varying the shadowing standard deviation $\sigma_{\mathrm{sh}}$, the path loss exponent $\gamma$, and the carrier frequency $f_{c}$, for $E_{b} / N_{0}=26 \mathrm{~dB}$ at the receiver side. The shadowing caused by the wireless channel produces large local-mean power variations. Hence, as expected, higher values of $\sigma_{\text {sh }}$ produce higher BERs, as can be noticed from Figure 21(a). In this figure, it can be seen that the 16FSK, the 64FSK and the 16QAM achieved the best performance under shadowing. The poorest performance was attained by the 64PSK.

The effect of different path loss exponents is shown in Figure 21(b), where it can be observed that this parameter has a lower impact on the BER, when compared with the shadowing. The poorest performance was attained by the 64PSK modulation, with the other ones performing relatively close to each other, again with the advantage of the 16FSK, the 64FSK and the 16QAM.

In what concerns different carrier frequencies, Figure 21(c) unveils a behavior that resembles the one depicted in Figure 21(a). Thus, the same conclusions apply.

\footnotetext{
${ }^{1}$ The $28 \mathrm{GHz}$ frequency is not formally in the mm-wave range, but it arises as a target for 5G applications [26], [28].
} 


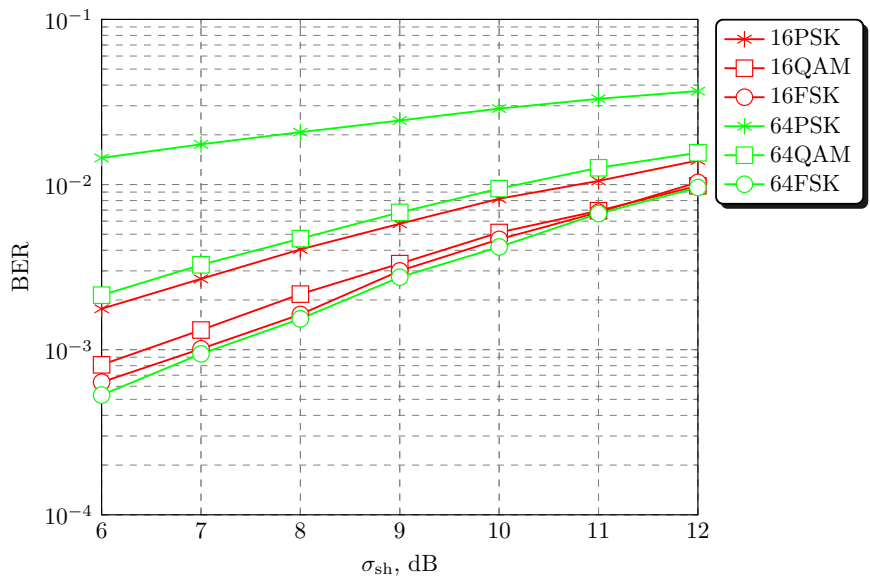

(a)

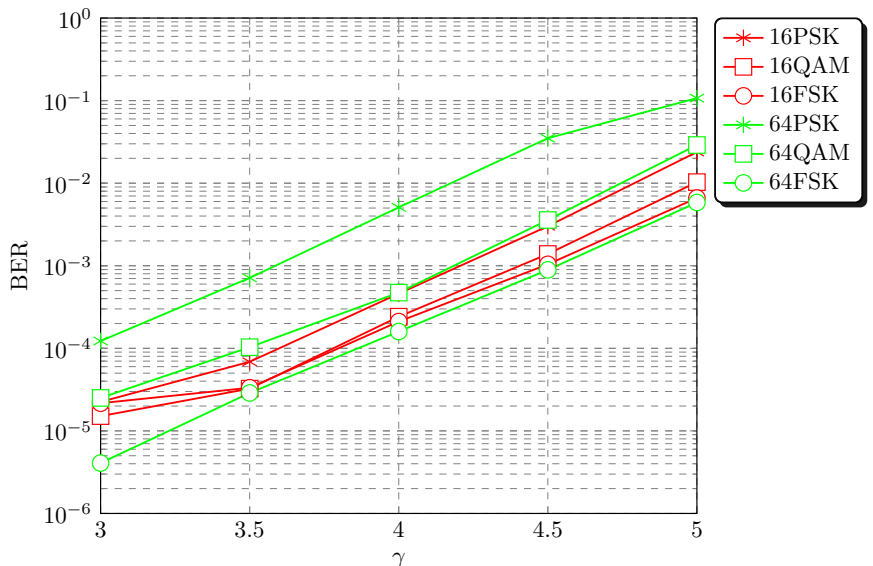

(b)

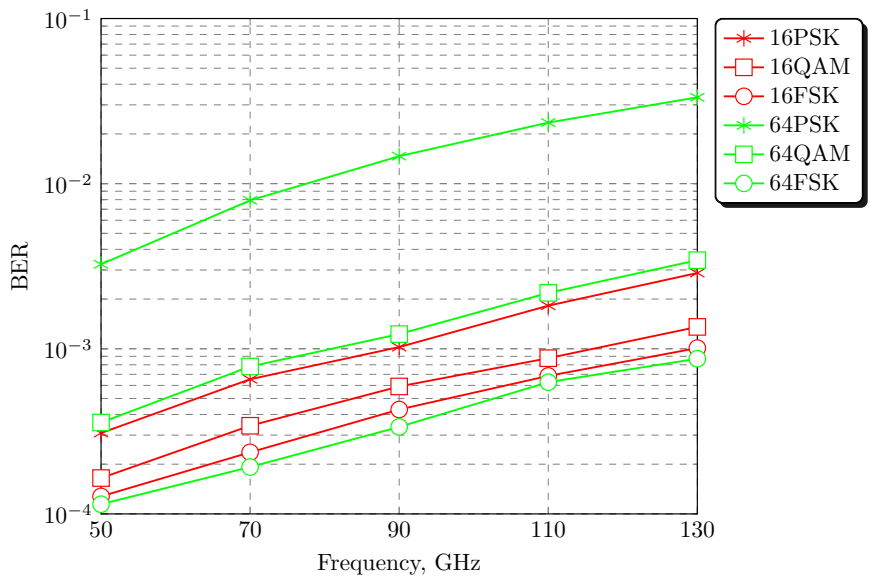

(c)

Fig. 21. BER versus (a) shadowing standard deviation $\sigma_{\mathrm{sh}}$, (b) path loss exponent $\gamma$, and (c) carrier frequency $f_{c}$, for $E_{b} / N_{0}=26 \mathrm{~dB}$ at the receiver.

\section{E. Joint Effect of the Impairments}

Finally, a performance analysis taking into account the aforementioned impairments altogether is made in this subsection. Figure 22 displays BER curves for all modulations in presence of phase noise with total variance given by $\sigma_{\mathrm{ph}}^{2}=1.345 \times 10^{-5} \mathrm{rad}^{2}$, I-Q imbalance factors $\rho=0.2$ and $\theta \stackrel{\circ}{=} 2^{\circ}$, and amplifier parameters $\delta=-6000$ and $V_{\text {sat }}=8$ volts. These values were adopted to yield an EVM of $8 \%$ for the 64QAM modulation, and correspond approximately to the middle of the scales when the individual impairments were analyzed. The 4PSK/4QAM modulation attained the smallest BER, even smaller than the 64FSK. This behavior can be confirmed by the individual impairment analysis presented in the previous subsections. The MFSK modulations keep maintaining their particular characteristic of BER reduction for higher $M$. In case of the 64PSK, one can notice an evident error floor and an error floor starting to show up in the case of the 64QAM modulation. These floors are mainly due to the phase noise influence; the results in Figures 15, 17, and 19 support for this conclusion. It is important to observe that, when using high-quality hardware, the 16QAM performance under all impairments is very similar to the ones attained by the MFSK modulations. Thus, it is viable to employ the 16QAM scheme on the mm-wave range, conflicting with the conclusions reported in [2]. The behavior unveiled in [2], where the MFSK attained a substantially lower BER than the 16QAM, is not consistent with the actual technology stage, meaning that a parameter oversizing was adopted in this reference.

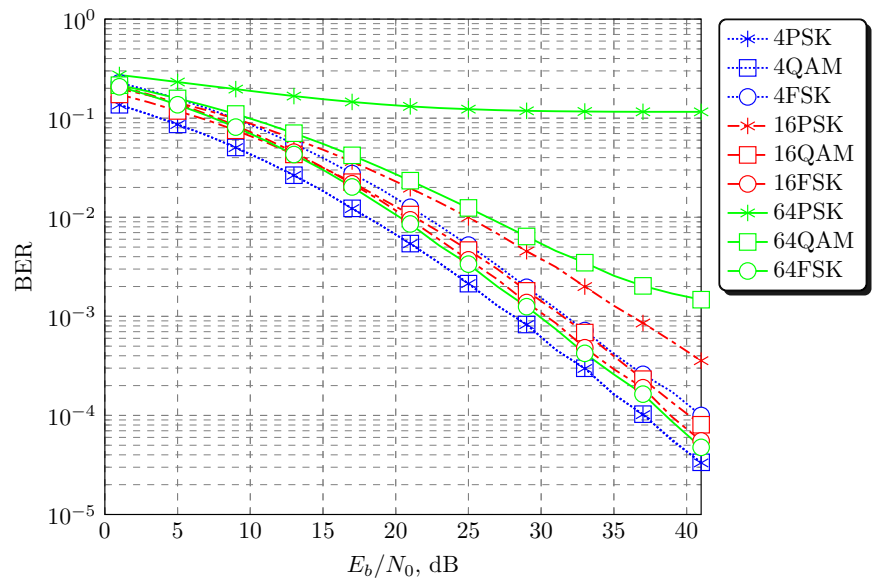

Fig. 22. BER versus $E_{b} / N_{0}$, for $\sigma_{\mathrm{ph}}^{2}=1.345 \times 10^{-5} \mathrm{rad}^{2}, \rho=0.2 \mathrm{~dB}$, and $\theta=2^{\circ}, V_{\text {sat }}=8 \mathrm{~V}$, and $\delta / 8=-6000, \sigma_{\text {sh }}=9 \mathrm{~dB}, \gamma=4$, and $f_{c}=60 \mathrm{GHz}$.

\section{CONCLUSIONS AND OPPORTUNITIES}

In this tutorial article, the theory about important hardware and channel impairments that might deeply affect the performance of wireless communication systems in the millimeter wave range was presented. This theory was then applied to the development of models for assessing the performance of digital modulations under the separate and under the joint effect of such impairments. The modulations analyzed were the $M$-ary frequency-shift keying with non-coherent detection, and the $M$-ary phase-shift keying and $M$-ary quadrature amplitude modulation with coherent detection.

Besides a comprehensive theoretical treatment, simulation results were given and brought out the different degrees of robustness of the above-mentioned modulations to the modeled impairments. It was unveiled that the MFSK is attractive for application in the millimeter wave range, but it is not always the unique robust solution, contrasting with recent claims in the literature. Along with the MFSK, the 16QAM modulation arises as interesting candidate for applications in this range. 
As an object of future research, others modulation schemes could be analyzed under the presented models in order to obtain a more generic conclusion about their potentials to be applicable with carrier frequencies in the millimeter wave range. Multicarrier modulation schemes could be included in this analysis, which motivates the nontrivial adaptation of the combined impairment model to a matrix form compatible with the multiple carrier system and with the frequency selectivity of the channel. Other particular impairments inherent to multicarrier signals could be considered as well.

\section{REFERENCES}

[1] A. Osseiran, F. Boccardi, V. Braun, K. Kusume, P. Marsch, M. Maternia, O. Queseth, M. Schellmann, H. Schotten, H. Taoka, H. Tullberg, M. A Uusitalo, B. Timus, and M. Fallgren, "Scenarios for $5 \mathrm{G}$ mobile and wireless communications: the vision of the METIS project," IEEE Communications Magazine, vol. 52, no. 5, pp. 26-35, May 2014, DOI: 10.1109/MCOM.2014.6815890.

[2] A. A. Nasir, H. Mehrpouyan, D. Matolak, and S. Durrani, "Noncoherent FSK: An attractive modulation set for millimeter-wave communications," in 2016 IEEE Wireless Communications and Networking Conference, April 2016, pp. 1-7, DOI: 10.1109/WCNC.2016.7565093.

[3] H. Mehrpouyan, M. R. Khanzadi, M. Matthaiou, A. M. Sayeed, R. Schober, and Y. Hua, "Improving bandwidth efficiency in E-band communication systems," IEEE Communications Magazine, vol. 52, no. 3, pp. 121-128, March 2014, DOI: 10.1109/MCOM.2014.6766096.

[4] T. Schenk, RF imperfections in high-rate wireless systems: impact and digital compensation. Springer Science \& Business Media, 2008, DOI: 10.1007/978-1-4020-6903-1.

[5] M. Matthaiou, A. Papadogiannis, E. Bjornson, and M. Debbah, "Twoway relaying under the presence of relay transceiver hardware impairments," IEEE Communications Letters, vol. 17, no. 6, pp. 1136-1139, June 2013, DOI: 10.1109/LCOMM.2013.042313.130191.

[6] D. A. Guimarães, Digital transmission: A simulation-aided introduction with VisSim/Comm. Springer Verlag, Berlin-Düsseldorf, 2009, DOI: 10.1007/978-3-642-01359-1.

[7] R. Zhou, Y. Zhang, W. Rhee, and Z. Wang, " $2.4 \mathrm{GHz} 20 \mathrm{Mb} / \mathrm{s}$ FSK receiver front-end and transmitter modulation PLL design for energyefficient short-range communicaiton," in 2016 IEEE International Conference on Electron Devices and Solid-State Circuits (EDSSC), Aug 2016, pp. 291-294, DOI: 10.1109/EDSSC.2016.7785265.

[8] K.-C. Huang and Z. Wang, Millimeter wave communication systems. John Wiley \& Sons, 2011, vol. 29, DOI: 10.1002/9780470889886.

[9] T. Reis and D. Guimarães, "Modelo para avaliação de modulações digitais na faixa de ondas milimétricas," in XXXV Simpósio Brasileiro de Telecomunicações e Processamento de Sinais (SBrT 2017), São Pedro, Brazil, Sep. 2017, pp. 851-855.

[10] M. R. Khanzadi, D. Kuylenstierna, A. Panahi, T. Eriksson, and H. Zirath, "Calculation of the performance of communication systems from measured oscillator phase noise," IEEE Transactions on Circuits and Systems I: Regular Papers, vol. 61, no. 5, pp. 1553-1565, May 2014, DOI: 10.1109/TCSI.2013.2285698.

[11] A. Hajimiri and T. H. Lee, "A general theory of phase noise in electrical oscillators," IEEE Journal of Solid-State Circuits, vol. 33, no. 2, pp. 179-194, Feb 1998, DOI: 10.1109/4.658619.

[12] H. Holma and A. Toskala, LTE for UMTS: Evolution to LTE-advanced. John Wiley \& Sons, 2011, DOI: 10.1002/9781119992943.

[13] A. Demir, A. Mehrotra, and J. Roychowdhury, "Phase noise in oscillators: a unifying theory and numerical methods for characterization," IEEE Transactions on Circuits and Systems I: Fundamental Theory and Applications, vol. 47, no. 5, pp. 655-674, May 2000, DOI: $10.1109 / 81.847872$

[14] A. Chorti and M. Brookes, "A spectral model for RF oscillators with power-law phase noise," IEEE Transactions on Circuits and Systems I: Regular Papers, vol. 53, no. 9, pp. 1989-1999, Sept 2006, DOI: 10.1109/TCSI.2006.881182

[15] M. R. Khanzadi, R. Krishnan, D. Kuylenstierna, and T. Eriksson, "Oscillator phase noise and small-scale channel fading in higher frequency bands," in 2014 IEEE Globecom Workshops (GC Wkshps), Dec 2014, pp. 410-415, DOI: 10.1109/GLOCOMW.2014.7063466.
[16] J. Antes and I. Kallfass, "Performance estimation for broadband multigigabit millimeter- and sub-millimeter-wave wireless communication links," IEEE Transactions on Microwave Theory and Techniques, vol. 63 , no. 10 , pp. 3288-3299, Oct 2015, DOI: 10.1109/TMTT.2015.2467390.

[17] N. J. Kasdin, "Discrete simulation of colored noise and stochastic processes and 1/f alpha; power law noise generation," Proceedings of the IEEE, vol. 83, no. 5, pp. 802-827, May 1995, DOI: 10.1109/5.381848.

[18] A. A. Abidi, "Direct-conversion radio transceivers for digital communications," IEEE Journal of Solid-State Circuits, vol. 30, no. 12, pp. 1399-1410, Dec 1995, DOI: 10.1109/4.482187.

[19] A. Baier, "Quadrature mixer imbalances in digital TDMA mobile radio receivers," in International Zurich Seminar on Digital Communications, Electronic Circuits and Systems for Communications., Mar 1990, pp. 147-162, DOI: 10.1109/DIGCOM.1990.129367.

[20] T. S. Rappaport, R. W. Heath Jr, R. C. Daniels, and J. N. Murdock, Millimeter wave wireless communications. Pearson Education, 2014, DOI: 10.1109/MWC.2015.7306370.

[21] C.-P. Liang, J.-H. Jong, W. E. Stark, and J. R. East, "Nonlinear amplifier effects in communications systems," IEEE Transactions on Microwave Theory and Techniques, vol. 47, no. 8, pp. 1461-1466, Aug 1999, DOI: $10.1109 / 22.780395$

[22] S. Cripps, RF power amplifiers for wireless communications. Artech House, 2006, DOI: 10.1109/MMW.2000.823830.

[23] H. Yang, "Towards low-cost gigabit wireless systems at $60 \mathrm{GHz}$," Ph.D. dissertation, Technische Universiteit Eindhoven, 2008, ISBN: 978-90386-1425-0.

[24] J. G. Proakis and M. Salehi, Communication systems engineering. Prentice Hall New Jersey, 2001, vol. 2, ISBN: 978-0130617934.

[25] S. Forestier, P. Bouysse, R. Quere, A. Mallet, J. M. Nebus, and L. Lapierre, "Joint optimization of the power-added efficiency and the error-vector measurement of 20-GHz pHEMT amplifier through a new dynamic bias-control method," IEEE Transactions on Microwave Theory and Techniques, vol. 52, no. 4, pp. 1132-1141, April 2004, DOI: 10.1109/TMTT.2004.825745.

[26] G. R. MacCartney, J. Zhang, S. Nie, and T. S. Rappaport, "Path loss models for 5G millimeter wave propagation channels in urban microcells," in 2013 IEEE Global Communications Conference (GLOBECOM), Dec 2013, pp. 3948-3953, DOI: 10.1109/GLOCOM.2013.6831690.

[27] Z. Chen and F. F. Dai, "Effects of LO phase and amplitude imbalances and phase noise on $M$-QAM transceiver performance," IEEE Transactions on Industrial Electronics, vol. 57, no. 5, pp. 1505-1517, May 2010, DOI: $10.1109 /$ TIE.2010.2042417

[28] G. R. Maccartney, T. S. Rappaport, S. Sun, and S. Deng, "Indoor office wideband millimeter-wave propagation measurements and channel models at 28 and $73 \mathrm{GHz}$ for ultra-dense $5 \mathrm{G}$ wireless networks," IEEE Access, vol. 3, pp. 2388-2424, 2015, DOI: 10.1109/ACCESS.2015.2486778.

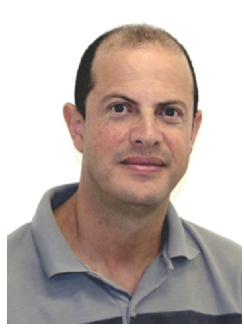

Dayan Adionel Guimarães received the Ph.D. degree in Electrical Engineering from the State University of Campinas, Unicamp, Brazil, in 2003. $\mathrm{He}$ is currently a Lecturer and a Researcher with the National Institute of Telecommunications, Inatel, Brazil. His research interests are the general aspects of fixed and mobile wireless communications, specifically radio propagation, digital transmission, spectrum sensing for cognitive radio, and convex optimization and signal processing applied to communications.

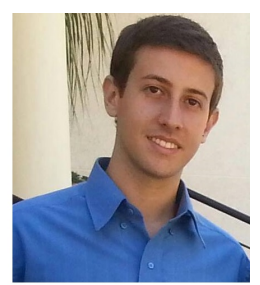

Tiago Magalhães received the B.Sc. and the M.Sc. degrees in Electrical Engineering from Inatel, Brazil, in 2015 and 2018, respectively. He worked with digital circuits development and microwave link design, and currently he is developing smartphones with the Samsumg-Sidia of Amazonas, Brazil. His main area of interest includes the general aspects of fixed and mobile wireless communications, specially digital transmission. 